\title{
Synthesis and biological evaluation of some 4-aminoquinoline derivatives as potential antitubercular agents
}

\author{
Shankar Alegaon ${ }^{1 *}$ (D), Kamlesh Kashniyal', Sanket Kuncolienkarr ${ }^{1}$, Rohini Kavalapure ${ }^{1}$, Preeti Salve ${ }^{1}$, Mahesh Palled ${ }^{1}$, \\ Shailendra Suryawanshi ${ }^{1}$ and Sunil Jalalpure ${ }^{2,3}$
}

\begin{abstract}
Background: Based on bioisosteric similarities with thiacetazone, a series of 7-chloro-4-aminoquinoline derivatives have been designed and synthesized. The target compounds were elucidated by NMR, mass, and FTIR spectral data. All synthesized compounds were evaluated for their in vitro antitubercular activity against Mycobacterium tuberculosis $\mathrm{H}_{37} \mathrm{Rv}(\mathrm{MTB})$, and human dermal fibroblast cell lines were used to assess toxicity of selected ligands.

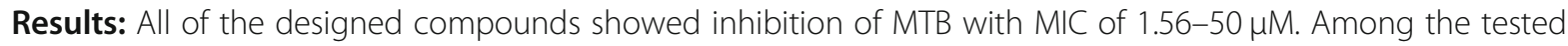
compounds, $7 \mathrm{c}$ and $7 \mathrm{~g}$ proved to be most potent MTB inhibitors ( $\mathrm{MIC}=1.56 \mu \mathrm{M})$.

Conclusions: The outcome of present study suggests that most of the synthesized compounds are sensitive to Mycobacterium tuberculosis and showed acceptable range for molecular parameters. Thus, 7-chloro-4aminoquinolines could be a useful lead for the development of new MTB inhibitory agents.
\end{abstract}

Keywords: Thiosemicarbazones, Semicarbazones, Quinoline, Antitubercular, Cytotoxicity

\section{Background}

Tuberculosis (TB) is considered to be the most widespread and lethal communicable disease in the world. TB is caused by an infection with the slow-growing Mycobacterium tuberculosis (MTB). According to the World Health Organization, there were an estimated 10.0 million new TB cases in 2017 with 1.3 million TB deaths. In addition, more than $65 \%$ of the world's TB cases occur in Southeast Asia, the West Pacific, and Africa [1]. Commonly used TB treatment regimen DOTS (directly observed therapy short-course) requires taking INH, PZA, and RIF for 60 days followed by an additional 120 days of treatment with INH and RIF $[2,3]$. Due to the resistance acquired by the Mycobacterium tuberculosis against various first-line drugs [4], there is a need to find new and better drug candidates for the treatment. Quinoline and analogs have always attracted the attention of industrial and academic personnel. It is

\footnotetext{
* Correspondence: sgalegaon@gmail.com

'Department of Pharmaceutical Chemistry, KLE College of Pharmacy, Belagavi, KLE Academy of Higher Education and Research, Belagavi, Karnataka 590 010, India

Full list of author information is available at the end of the article
}

being reported as having many interesting biological properties such as antimalarial $[5,6]$, cytotoxic $[7,8]$, anti-inflammatory $[9,10]$, antiviral $[11,12]$, antimicrobial [13, 14], antifungal [15], anti-Alzheimer [16], antiprotozoal [17], and antitubercular [18] activities. Quinoline is a basic pharmacophore used for the design of antitubercular agents, like mefloquine, ciprofloxacin, moxifloxacin, and bedaquiline which are already in the market [18]. Furthermore, thiosemicarbazone and semicarbazone derivatives are frequently used scaffolds in biology-orientated synthesis which possess a huge variety of pharmacological activities including an antibacterial, antifungal, antitubercular, and anticancer activity $[19$, 20].

In view of the above observations, the diverse pharmacological activities of these pharmacophores prompted us to synthesize the target compounds, presuming that their hybrid in a one-structural entity could potentially yield new heterocyclic compounds with significant synergistic antitubercular properties. Hence, with an aim to develop promising antitubercular agents, a series of novel 7-chloro-N-phenylquinolin-4-amine derivatives 


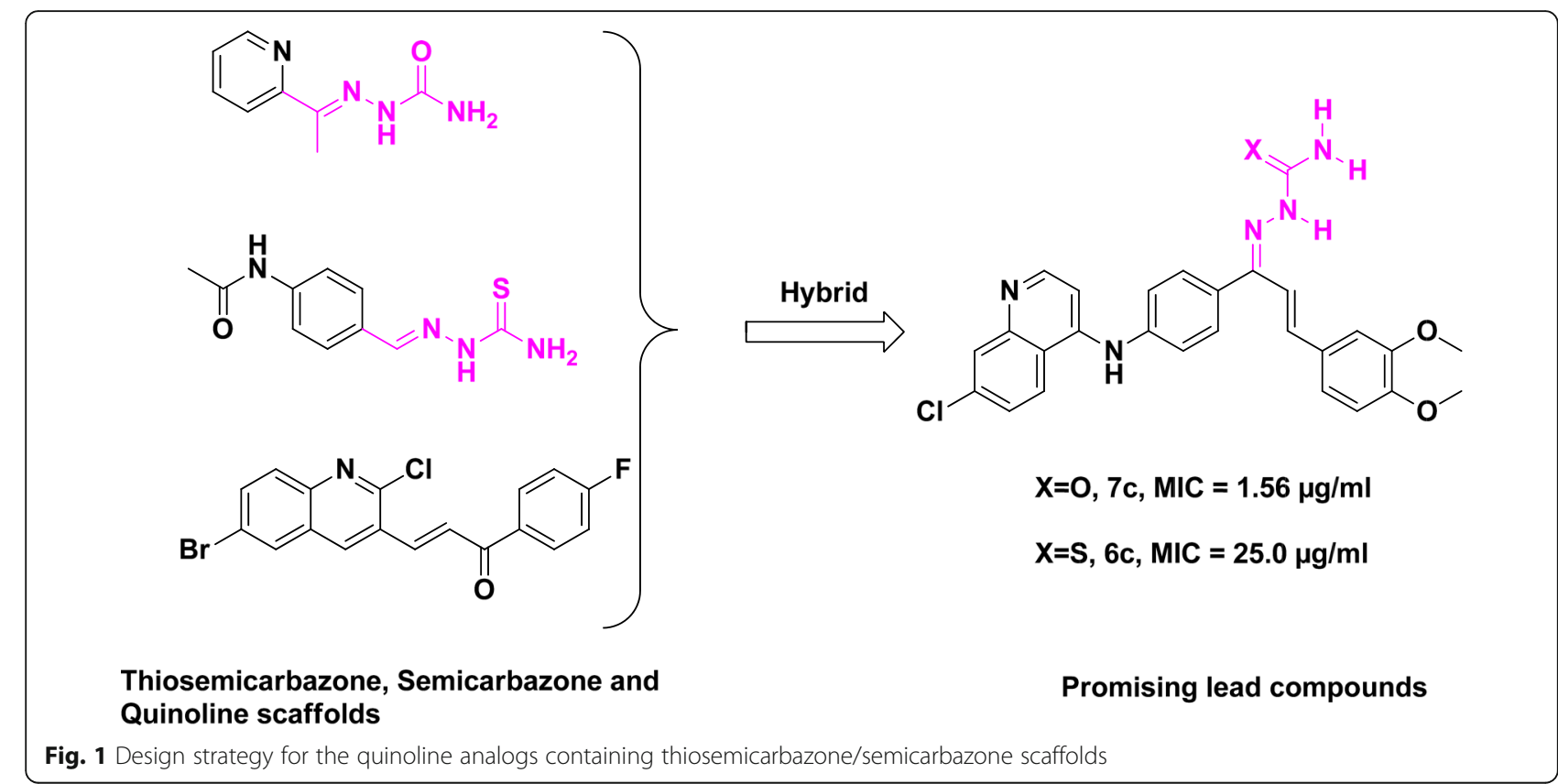

containing thiosemicarbazone and semicarbazone [19, 20], scaffolds were designed and synthesized (Fig. 1). In the present study, all target ligands are screened for their in vitro antitubercular activity against Mycobacterium tuberculosis $\mathrm{H}_{37} \mathrm{Rv}$ (MTB), and human dermal fibroblast cell line were used for toxicity study.

\section{Method}

Synthesis of thiosemicarbazide and semicarbazide derivatives

An equimolar amount of thiosemicarbazide or semicarbazide hydrochloride $(20 \mathrm{mmol})$ and substituted $\alpha, \beta$-unsaturated ketones (4a-i, and 5a-i) (20 mmol) [21] were dissolved in isopropyl alcohol, and glacial acetic acid $(0.1 \mathrm{ml})$ was used as a catalyst. The mixture was stirred at $85{ }^{\circ} \mathrm{C}$ for $36 \mathrm{~h}$. On completion of the reaction, as monitored by TLC, the reaction mixture was cooled. Precipitate was filtered by suction and washed with cooled isopropyl alcohol and solvent ether. The structures of target compounds were established by using different spectroscopic techniques.

\section{Antitubercular activity assay}

Microplate alamar blue assay (MABA) method [22, 23] was adopted to evaluate antitubercular activities (Additional file 1).

\section{Cytotoxic assay}

MTT (3-(4, 5-dimethylthiazo-2-yl)-2,5-diphenyl-tetrazolium bromide) [24] assay protocol was used to assess the toxicity studies of selected analogs.

\section{Results}

Synthesis and spectral data

((E)-1-(4-((7-chloroquinolin-4-yl)amino)phenyl)-3-(ptolyl)allylidene)hydrazinecarbothioamide(6a)

Yield: 55\%; m.p.: $196-198^{\circ} \mathrm{C}$; IR $\left(\mathrm{KBr} \mathrm{cm}{ }^{-1}\right)$ : 3362 $\left(\mathrm{NH}_{2}\right), 1593(\mathrm{C}=\mathrm{C}), 1351(\mathrm{C}=\mathrm{S}), 1170\left(\mathrm{OCH}_{3}\right) ;{ }^{1} \mathrm{H}-\mathrm{NMR}$ $\left(400 \mathrm{MHz}, \mathrm{DMSO}-d_{6}\right) \delta(\mathrm{ppm})=2.62\left(\mathrm{~s}, 3 \mathrm{H}, \mathrm{CH}_{3}\right), 7.05$ (s, 1H, ArH), 7.37 (d, 2H, J=8.4 Hz, ArH), $7.56(\mathrm{~m}, 4 \mathrm{H}$, $\mathrm{ArH}$ ), 7.72-7.69 (m, 3H, ArH, -C=HC-), 7.83 (d, 1H, $J=$ $15.6 \mathrm{~Hz},-\mathrm{C}=\mathrm{HC}-), 7.85$ (s, $\left.1 \mathrm{H}, \mathrm{NH}_{2}\right), 7.88\left(\mathrm{~s}, 1 \mathrm{H}, \mathrm{NH}_{2}\right)$, 7.91 (s, 1H, ArH), 8.18 (s, 1H, ArH), 8.42 (s, 1H, ArH), 8.55 (s, 1H, ArH), 9.95 (s, 1H, NH), 10.83 (s, 1H, =N$\mathrm{NH}-\mathrm{C}) ; \mathrm{LC}-\mathrm{MS} M / Z: 473[\mathrm{M}+\mathrm{H}]^{+}$.

2-((E)-1-(4-((7-chloroquinolin-4-yl)amino)phenyl)-3-(4methoxyphenyl)allylidene)hydrazinecarbothioamide(6b)

Yield: 53\%; m.p.: $190-192{ }^{\circ} \mathrm{C}$; IR $\left(\mathrm{KBr} \mathrm{cm} \mathrm{cm}^{-1}\right): 3367$ $\left(\mathrm{NH}_{2}\right), 1593(\mathrm{C}=\mathrm{C}), 1350(\mathrm{C}=\mathrm{S}), 1172\left(\mathrm{OCH}_{3}\right) ;{ }^{1} \mathrm{H}-\mathrm{NMR}$ $\left(400 \mathrm{MHz}, \mathrm{DMSO}-d_{6}\right) \delta(\mathrm{ppm}) 3.82\left(\mathrm{~s}, 3 \mathrm{H}, \mathrm{OCH}_{3}\right), 7.02$ (s, 1H, ArH), $7.34(\mathrm{~d}, 2 \mathrm{H}, J=8.4 \mathrm{~Hz}, \operatorname{ArH}), 7.53(\mathrm{~m}, 4 \mathrm{H}$, ArH), 7.70-7.63 (m, 3H, ArH, -C=HC-), 7.85 (d, 1H, J= $15.6 \mathrm{~Hz},-\mathrm{C}=\mathrm{HC}-), 7.87$ (s, $\left.1 \mathrm{H}, \mathrm{NH}_{2}\right), 7.89$ (s, $\left.1 \mathrm{H}, \mathrm{NH}_{2}\right)$, 7.88 (s, 1H, ArH), 8.18 (s, 1H, ArH), 8.40 (s, 1H, ArH), 8.58 (s, 1H, ArH), $9.93(\mathrm{~s}, 1 \mathrm{H}, \mathrm{NH}), 10.81(\mathrm{~s}, 1 \mathrm{H},=\mathrm{N}$ $\mathrm{NH}-\mathrm{C})$; LC-MS $M / Z: 489[\mathrm{M}+\mathrm{H}]^{+}$.

2-((E)-1-(4-((7-chloroquinolin-4-yl)amino)phenyl)-3-(3,4dimethoxyphenyl)allylidene)hydrazinecarbothioamide(6c)

Yield: $56 \%$; m.p.: $148-150{ }^{\circ} \mathrm{C}$; IR $\left(\mathrm{KBr} \mathrm{cm}^{-1}\right)$ : 3367 $\left(\mathrm{NH}_{2}\right), 1265\left(\mathrm{OCH}_{3}\right), 1350(\mathrm{C}=\mathrm{S}) ;{ }^{1} \mathrm{H}-\mathrm{NMR}(400 \mathrm{MHz}$, DMSO- $\left.d_{6}\right) \delta(\mathrm{ppm})=3.81\left(\mathrm{~s}, 3 \mathrm{H}, \mathrm{OCH}_{3}\right), 3.86(\mathrm{~s}, 3 \mathrm{H}$, $\left.-\mathrm{OCH}_{3}\right), 7.02$ (s, 1H, ArH), 7.45 (s, 1H, ArH), 7.38 (s, 
1H, ArH), $7.53(\mathrm{~m}, 3 \mathrm{H}, \mathrm{ArH}), 7.72-7.69(\mathrm{~m}, 3 \mathrm{H}, \mathrm{ArH}$, -C=HC-), 7.85 (d, 1H, J=15.6 Hz, -C=HC-), $7.91(\mathrm{~s}, 1 \mathrm{H}$, $\mathrm{NH}_{2}$ ), 7.96 (s, 1H, $\mathrm{NH}_{2}$ ), 7.98 (s, 1H, ArH), 8.23 (s, 1H, ArH), 8.47 (s, 1H, ArH), 8.61 (s,1H, ArH), 9.91 (s, 1H, $\mathrm{NH}), 10.82$ (s, 1H, =N-NH-C); LC-MS M/Z: $519[\mathrm{M}+$ $\mathrm{H}]^{+}$.

2-((E)-1-(4-((7-chloroquinolin-4-yl)amino)phenyl)-3-(3,4,5trimethoxyphenyl)allylidene)hydrazinecarbothioamide(6d) Yield: $66 \%$; m.p.: $138-140{ }^{\circ} \mathrm{C}$; IR $\left(\mathrm{KBr} \mathrm{cm}^{-1}\right)$ : 3343 $\left(\mathrm{NH}_{2}\right), 1593(\mathrm{C}=\mathrm{C}), 1352(\mathrm{C}=\mathrm{S}), 1128\left(\mathrm{OCH}_{3}\right), 1128$ $\left(\mathrm{OCH}_{3}\right) ;{ }^{1} \mathrm{H}-\mathrm{NMR}\left(400 \mathrm{MHz}, \mathrm{DMSO}-d_{6}\right) \delta(\mathrm{ppm})=3.76$ (s, $\left.3 \mathrm{H}, \mathrm{OCH}_{3}\right), 3.86\left(\mathrm{~s}, 6 \mathrm{H},-\mathrm{OCH}_{3}\right), 6.42$ (s, 1H, ArH), 7.27 (d, 2H, J=8.4 Hz ArH), 7.42 (s, 1H, ArH), 7.52 (s, $1 \mathrm{H}, \mathrm{ArH}), 7.71-7.64$ (m, 3H, ArH, $-\mathrm{C}=\mathrm{HC}-), 7.89$ (d, 1H, $J=15.6 \mathrm{~Hz},-\mathrm{C}=\mathrm{HC}-), 7.92\left(\mathrm{~s}, 1 \mathrm{H}, \mathrm{NH}_{2}\right), 7.95(\mathrm{~s}, 1 \mathrm{H}$, $\mathrm{NH}_{2}$ ), 7.98(s, 1H, ArH), 8.23 (s, 1H, ArH), 8.40 (s, 1H, ArH), 8.59 (s, 1H, ArH), 9.68 (s, 1H, NH), 10.72 (s, 1H, =N-NH-C); LC-MS M/Z: $549[\mathrm{M}+\mathrm{H}]^{+}$.

\section{2-((E)-3-(4-chlorophenyl)-1-(4-((7-chloroquinolin-4-} yl)amino)phenyl)allylidene)hydrazinecarbothioamide(6e)

Yield: $73 \%$; m.p.: $142-144^{\circ} \mathrm{C}$; IR $\left(\mathrm{KBr} \mathrm{cm} \mathrm{cm}^{-1}\right): 3365$ $\left(\mathrm{NH}_{2}\right), 1554(\mathrm{C}=\mathrm{C}), 1350(\mathrm{C}=\mathrm{S}) ;{ }^{1} \mathrm{H}-\mathrm{NMR}(400 \mathrm{MHz}$, DMSO- $\left.d_{6}\right) \delta(\mathrm{ppm})=7.08(\mathrm{~s}, 1 \mathrm{H}, \mathrm{ArH}), 7.51-7.749(\mathrm{~m}$, $3 \mathrm{H}, \mathrm{ArH},-\mathrm{C}=\mathrm{HC}-$ ), 7.62 (d, 2H, $J=8.8 \mathrm{~Hz}, \mathrm{ArH}), 7.70$ $(\mathrm{d}, 1 \mathrm{H}, J=15.6 \mathrm{~Hz}-\mathrm{C}=\mathrm{HC}-), 7.78(\mathrm{~s}, 1 \mathrm{H}, \mathrm{ArH}), 7.89$ (s, $1 \mathrm{H}, \mathrm{NH}_{2}$ ), $7.91\left(\mathrm{~s}, 1 \mathrm{H}, \mathrm{NH}_{2}\right), 7.95$ (s, 1H, ArH), 8.01 (d, $2 \mathrm{H}, J=8.4 \mathrm{~Hz}, \mathrm{ArH}), 8.20$ (d, $2 \mathrm{H}, J=8.8 \mathrm{~Hz}, \mathrm{ArH}), 8.41$ (s, 1H, ArH), 8.62 (s, 1H, ArH), 10.08 (s, 1H, NH), 11.06 (s, $1 \mathrm{H},=\mathrm{N}-\mathrm{NH}-\mathrm{C})$; LC-MS $M / Z: 494[\mathrm{M}+\mathrm{H}]^{+}$.

\section{2-((E)-1-(4-((7-chloroquinolin-4-yl)amino)phenyl)-3-(4-}

\section{fluorophenyl)allylidene)hydrazinecarbothioamide(6f)}

Yield: $58 \%$; m.p.: $178-180{ }^{\circ} \mathrm{C}$; IR $\left(\mathrm{KBr} \mathrm{cm}^{-1}\right)$ : 3354 $\left(\mathrm{NH}_{2}\right), 1575(\mathrm{C}=\mathrm{C}), 1351(\mathrm{C}=\mathrm{S}) ;{ }^{1} \mathrm{H}-\mathrm{NMR}(400 \mathrm{MHz}$, DMSO- $\left.d_{6}\right) \delta(\mathrm{ppm})=7.01(\mathrm{~s}, 1 \mathrm{H}, \mathrm{ArH}), 7.32-7.24(\mathrm{~m}$, $3 \mathrm{H}, \mathrm{ArH},-\mathrm{C}=\mathrm{HC}-$ ), 7.52 (d, 2H, $J=8.8 \mathrm{~Hz}, \mathrm{ArH}), 7.64$ $(\mathrm{d}, 1 \mathrm{H}, J=15.6 \mathrm{~Hz}-\mathrm{C}=\mathrm{HC}-), 7.66\left(\mathrm{~s}, 1 \mathrm{H}, \mathrm{NH}_{2}\right), 7.75(\mathrm{~s}$, $1 \mathrm{H}, \mathrm{NH}_{2}$ ), 7.98 (s, 1H, ArH), 7.98-7.95 (m, 3H, ArH), 8.23-8.20 (m, 2H, ArH), 8.46 (s, 1H, ArH), 8.60 (s, 1H, ArH), 10.02 (s, 1H, NH), 11.03 (s, 1H, =N-NH-C); LCMS $M / Z: 477[\mathrm{M}+\mathrm{H}]^{+}$.

\section{2-((E)-3-(4-bromophenyl)-1-(4-((7-chloroquinolin-4-} yl)amino)phenyl)allylidene)hydrazinecarbothioamide(6 g)

Yield: $66 \%$; m.p.: $186-188^{\circ} \mathrm{C}$; IR $\left(\mathrm{KBr} \mathrm{cm}^{-1}\right)$ : 3375 $\left(\mathrm{NH}_{2}\right), 1580(\mathrm{C}=\mathrm{C}), 1352(\mathrm{C}=\mathrm{S}) ;{ }^{1} \mathrm{H}-\mathrm{NMR}(400 \mathrm{MHz}$, DMSO- $\left.d_{6}\right) \delta(\mathrm{ppm})=6.53(\mathrm{~s}, 1 \mathrm{H}, \mathrm{ArH}), 6.86(\mathrm{~d}, 1 \mathrm{H}, J=$ $15.6 \mathrm{~Hz},-\mathrm{C}=\mathrm{HC}-), 6.99$ (d, $1 \mathrm{H}, J=12.6 \mathrm{~Hz},-\mathrm{C}=\mathrm{HC}-$ ), 7.07 (s, 1H, ArH), 7.30 (s, 1H, ArH), 7.47 (s, 1H, ArH), 7.62 (s, 1H, $\mathrm{NH}_{2}$ ), 7.77-7.70 (m, 4H, ArH,), 7.81 (s, 1H, $\left.\mathrm{NH}_{2}\right) 7.85$ (d, 2H, $\left.J=8.4 \mathrm{~Hz}, \operatorname{ArH}\right), 8.20$ (s, 1H, ArH),
8.30 (s, 1H, ArH), 8.51 (s, 1H, ArH), 10.10 (s, 1H, NH), 11.07 (s, 1H, =N-NH-C); LC-MS $M / Z: 538[\mathrm{M}+\mathrm{H}]^{+}$.

2-((E)-1-(4-((7-chloroquinolin-4-yl)amino)phenyl)-3-(4nitrophenyl)allylidene)hydrazinecarbothioamide( $6 h$ )

Yield: $47 \%$; m.p.: $216-218^{\circ} \mathrm{C}$; IR $\left(\mathrm{KBr} \mathrm{cm}^{-1}\right)$ : 3361 $\left(\mathrm{NH}_{2}\right), 1540(\mathrm{C}=\mathrm{C}), 1350(\mathrm{C}=\mathrm{S})$; ${ }^{1} \mathrm{H}-\mathrm{NMR} \quad(400 \mathrm{MHz}$, DMSO- $\left.d_{6}\right) \delta(\mathrm{ppm})=7.20(\mathrm{~d}, 1 \mathrm{H}, J=5.6 \mathrm{~Hz}, \mathrm{ArH}), 7.23-$ 7.10 (m, 4H, ArH, $\mathrm{NH}_{2}$ ), 7.47 (d, 2H, $\left.J=8.8 \mathrm{~Hz}, \mathrm{ArH}\right)$, 7.71-7.60 (m, 3H, ArH, -C=HC-), 7.96 (s, 1H, NH $\mathrm{NH}_{2}$, 8.13 (s, 1H, ArH), 8.26 (s, 1H, ArH), 8.44-8.38 (m, 4H, ArH), 9.87 (s, 1H, NH), 11.69 (s, 1H, =N-NH-C); LC-MS $M / Z: 504[\mathrm{M}+\mathrm{H}]^{+}$.

2-((E)-1-(4-((7-chloroquinolin-4-yl)amino)phenyl)-3-(4hydroxyphenyl)allylidene)hydrazinecarbothioamide(6i) Yield: 50\%; m.p.: $230-232{ }^{\circ} \mathrm{C}$; IR $\left(\mathrm{KBr} \mathrm{cm}^{-1}\right): 3365$ $\left(\mathrm{NH}_{2}\right), 1567(\mathrm{C}=\mathrm{C}), 1353(\mathrm{C}=\mathrm{S}) ;{ }^{1} \mathrm{H}-\mathrm{NMR}(400 \mathrm{MHz}$, DMSO- $\left.d_{6}\right) \delta(\mathrm{ppm})=7.20(\mathrm{~s}, 1 \mathrm{H}, \mathrm{ArH}), 7.27(\mathrm{~d}, 2 \mathrm{H}, J=$ 8.8 Hz, ArH), 7.45-7.40 (m, 3H, ArH, CH=C), 7.73-7.61 $(\mathrm{m}, 3 \mathrm{H}, \mathrm{ArH},-\mathrm{C}=\mathrm{HC}-), 7.93\left(\mathrm{~s}, 1 \mathrm{H}, \mathrm{NH}_{2}\right), 7.96(\mathrm{~s}, 1 \mathrm{H}$, $\mathrm{NH}_{2}$ ), 8.13 (s, 1H, ArH), 8.25 (d, 1H, ArH), 8.44-8.38 (m, 4H, ArH), 9.86 (s, 1H, NH), 9.88 (s, 1H, OH), 11.55 (s, $1 \mathrm{H},=\mathrm{N}-\mathrm{NH}-\mathrm{C})$; LC-MS $M / Z: 475[\mathrm{M}+\mathrm{H}]^{+}$.

\section{2-((E)-1-(4-((7-chloroquinolin-4-yl)amino)phenyl)-3-(p- tolyl)allylidene)hydrazinecarboxamide(7a)}

Yield: $72 \%$; m.p.: $148-150{ }^{\circ} \mathrm{C}$; IR $\left(\mathrm{KBr} \mathrm{cm}^{-1}\right): 3425$ $\left(\mathrm{NH}_{2}\right), 1693(\mathrm{C}=\mathrm{O}) ;{ }^{1} \mathrm{H}-\mathrm{NMR}\left(400 \mathrm{MHz}, \mathrm{DMSO}-d_{6}\right) \delta$ (ppm) $2.88\left(\mathrm{~s}, 3 \mathrm{H}, \mathrm{CH}_{3}\right), 6.51\left(\mathrm{~s}, 2 \mathrm{H}, \mathrm{NH}_{2}\right), 6.83-6.81$ (d, $2 \mathrm{H}, J=8.8 \mathrm{~Hz}, \mathrm{ArH}), 6.86(\mathrm{~s}, 1 \mathrm{H}, \mathrm{ArH}), 6.90(\mathrm{~s}, 1 \mathrm{H}$, $\operatorname{ArH}), 7.60$ (d, 2H, $J=8.8, \operatorname{ArH}), 7.73(\mathrm{~d}, 1 \mathrm{H}, J=15.6 \mathrm{~Hz}$, -C=HC-), 7.83 (d, 1H, J=15.6 Hz, -C=HC-), 7.85 (s, 1H, ArH), 7.93 (d, 2H, $J=8.4 \mathrm{~Hz}, \operatorname{ArH}), 8.06$ (d, 2H, $J=8.4$ $\mathrm{Hz}, \mathrm{ArH}), 8.57$ (s, 1H, ArH), 8.69 (s, 1H, ArH), 9.41 (s, $1 \mathrm{H}, \mathrm{NH}), 10.91(\mathrm{~s}, 1 \mathrm{H},=\mathrm{N}-\mathrm{NH}-\mathrm{C})$; LC-MS $M / Z: 457$ $[\mathrm{M}+\mathrm{H}]^{+}$.

2-((E)-1-(4-((7-chloroquinolin-4-yl)amino)phenyl)-3-(4methoxyphenyl)allylidene)hydrazinecarboxamide(7b)

Yield: 65\%; m.p.: $238-240{ }^{\circ} \mathrm{C}$; FTIR (KBr) cm $\mathrm{cm}^{-1}$ : 3450 $\left(\mathrm{NH}_{2}\right), 1685(\mathrm{C}=\mathrm{O}), 1251\left(\mathrm{OCH}_{3}\right) ;{ }^{1} \mathrm{H}-\mathrm{NMR}(400 \mathrm{MHz}$, DMSO- $\left.d_{6}\right) \delta(\mathrm{ppm})=3.72\left(\mathrm{~s}, 3 \mathrm{H}, \mathrm{OCH}_{3}\right), 6.48(\mathrm{~s}, 2 \mathrm{H}$, $\mathrm{NH}_{2}$ ), 6.88 (d, 2H,J=8.8 Hz, ArH), 6.96 (s, 1H, ArH), $7.13(\mathrm{~d}, 2 \mathrm{H}, J=8.8, \mathrm{ArH}), 7.51(\mathrm{~d}, 2 \mathrm{H}, J=8.4 \mathrm{~Hz}, \mathrm{ArH})$, $7.75(\mathrm{~d}, 1 \mathrm{H}, J=15.6 \mathrm{~Hz},-\mathrm{C}=\mathrm{HC}-), 7.78$ (d, 1H, $J=15.6$ $\mathrm{Hz},-\mathrm{C}=\mathrm{HC}-$ ), 7.85 (s, 1H, ArH), 7.93 (d, 2H, $J=8.8 \mathrm{~Hz}$, ArH), 8.0 (s, 1H, ArH), 8.54 (s, 1H, ArH), 8.72 (s, 1H, ArH), 9.38 (s, 1H, NH), 10.76 (s, 1H, =N-NH-C); LC-MS $M / Z: 473[\mathrm{M}+\mathrm{H}]^{+}$. 
2-((E)-1-(4-((7-chloroquinolin-4-yl)amino)phenyl)-3-(3,4dimethoxyphenyl)allylidene)hydrazinecarboxamide(7c) Yield: 56\%; m.p.: $214-216^{\circ} \mathrm{C}$; IR ( $\left.\mathrm{KBr} \mathrm{cm} \mathrm{cm}^{-1}\right)$ : 3442 $\left(\mathrm{NH}_{2}\right), 1681(\mathrm{C}=\mathrm{O}), 1255\left(\mathrm{OCH}_{3}\right) ;{ }^{1} \mathrm{H}-\mathrm{NMR}(400 \mathrm{MHz}$, $\left.\mathrm{DMSO}-d_{6}\right) \delta(\mathrm{ppm})=3.72\left(\mathrm{~s}, 3 \mathrm{H}, \mathrm{OCH}_{3}\right), 3.81(\mathrm{~s}, 3 \mathrm{H}$, $\left.-\mathrm{OCH}_{3}\right), 6.50$ (s, 2H, NH $\left.\mathrm{N}_{2}\right), 6.81$ (s, 1H, ArH), 7.20-6.96 (m, 3H, $J=8.4, A r H), 7.53(\mathrm{~d}, 2 \mathrm{H}, J=8.8 \mathrm{~Hz}, \mathrm{ArH}), 7.79$ (d, $1 \mathrm{H}, J=15.6 \mathrm{~Hz},-\mathrm{C}=\mathrm{HC}-), 7.81(\mathrm{~d}, 1 \mathrm{H}, J=15.6 \mathrm{~Hz}$, $-\mathrm{C}=\mathrm{HC}-), 7.85(\mathrm{~s}, 1 \mathrm{H}, \mathrm{ArH}), 7.95(\mathrm{~d}, 2 \mathrm{H}, J=8.4 \mathrm{~Hz}$, ArH), 8.07 (s, 1H, ArH), 8.56 (s, 1H, ArH), 8.72 (s, 1H, ArH), 10.20 (s, 1H, NH), 10.81 (s, 1H, =N-NH-C); LCMS $M / Z: 503[\mathrm{M}+\mathrm{H}]^{+}$.

\section{2-((E)-1-(4-((7-chloroquinolin-4-yl)amino)phenyl)-3-(3,4,5-} trimethoxyphenyl)allylidene)hydrazinecarboxamide(7d)

Yield: 52\%; m.p.: $238-240{ }^{\circ} \mathrm{C}$; IR $\left(\mathrm{KBr} \mathrm{cm}^{-1}\right)$ : 3442 $\left(\mathrm{NH}_{2}\right), 1671(\mathrm{C}=\mathrm{O}), 1251\left(\mathrm{OCH}_{3}\right) ;{ }^{1} \mathrm{H}-\mathrm{NMR}(400 \mathrm{MHz}$, DMSO- $\left.d_{6}\right) \delta(\mathrm{ppm})=3.82\left(\mathrm{~s}, 6 \mathrm{H}, \mathrm{OCH}_{3}\right), 3.87(\mathrm{~s}, 3 \mathrm{H}$, $\left.\mathrm{OCH}_{3}\right), 6.49$ (s, 2H, NH$\left.)_{2}\right), 6.76(\mathrm{~s}, 1 \mathrm{H}, \mathrm{ArH}), 6.88(\mathrm{~s}, 1 \mathrm{H}$, ArH), 7.54 (d, 2H, J=8.8 Hz, ArH), 7.71 (s, 1H, ArH), $7.81(\mathrm{~d}, 1 \mathrm{H}, J=15.6 \mathrm{~Hz},-\mathrm{C}=\mathrm{HC}-), 7.83(\mathrm{~d}, 1 \mathrm{H}, J=15.6$ $\mathrm{Hz},-\mathrm{C}=\mathrm{HC}-), 7.93(\mathrm{~d}, 2 \mathrm{H}, J=8.8, \mathrm{ArH}), 8.02(\mathrm{~s}, 2 \mathrm{H}$, ArH), 8.54 (s, 1H, ArH), 8.76 (s, 1H, ArH), 10.09 (s, 1H, $\mathrm{NH}), 11.02$ (s, 1H, =N-NH-C); LC-MS $M / Z: 533[\mathrm{M}+$ $\mathrm{H}]^{+}$.

\section{2-((E)-3-(4-chlorophenyl)-1-(4-((7-chloroquinolin-4-} yl)amino)phenyl)allylidene)hydrazinecarboxamide(7e)

Yield: $45 \%$; m.p.: $174-176{ }^{\circ} \mathrm{C}$; IR $\left(\mathrm{KBr} \mathrm{cm}^{-1}\right)$ : $3335\left(\mathrm{NH}_{2}\right.$, ), $1680(\mathrm{C}=\mathrm{O}), 1226(\mathrm{C}-\mathrm{N})$; ${ }^{1} \mathrm{H}-\mathrm{NMR}(400 \mathrm{MHz}, \mathrm{DMSO}-$ $\left.d_{6}\right) \delta(\mathrm{ppm})=6.48\left(\mathrm{~s}, 2 \mathrm{H}, \mathrm{NH}_{2}\right), 7.22(\mathrm{~s}, 1 \mathrm{H}, \mathrm{Ar}-\mathrm{H}), 7.54$ (d, $2 \mathrm{H}, J=8.4, \mathrm{Ar}-\mathrm{H}), 7.62(\mathrm{~d}, 2 \mathrm{H}, J=8.4 \mathrm{~Hz}, \mathrm{Ar}-\mathrm{H}), 7.76$ (s, 1H, Ar-H), 7.87 (d, 1H, J=15.6 Hz, $-\mathrm{CH}=\mathrm{C}), 7.89$ (d, $1 \mathrm{H}, J=15.6 \mathrm{~Hz},-\mathrm{CH}=\mathrm{C}), 7.95(\mathrm{~d}, 2 \mathrm{H}, J=8.8, \mathrm{Ar}-\mathrm{H})$, 7.99 (s, 1H, Ar-H), 8.05 (s, 1H, Ar-H), 8.29 (d, 2H, J= $8.8 \mathrm{~Hz}, \mathrm{Ar}-\mathrm{H}), 8.62$ (s, 1H, Ar-H), 10.17 (s, 1H, NH), $10.76(\mathrm{~s}, 1 \mathrm{H},=\mathrm{N}-\mathrm{NH}-\mathrm{C})$; LC-MS $M / Z: 477[\mathrm{M}+\mathrm{H}]^{+}$.

\section{2-((E)-1-(4-((7-chloroquinolin-4-yl)amino)phenyl)-3-(4-} fluorophenyl)allylidene)hydrazinecarboxamide(7f)

Yield: $70 \%$; m.p.: $230-232^{\circ} \mathrm{C}$; IR ( $\left.\mathrm{KBr} \mathrm{cm}^{-1}\right)$ : 3433 $\left(\mathrm{NH}_{2}\right), 1686(\mathrm{C}=\mathrm{O}), 1370(\mathrm{C}-\mathrm{N})$; ${ }^{1} \mathrm{H}-\mathrm{NMR}(400 \mathrm{MHz}$, DMSO- $\left.d_{6}\right) \delta(\mathrm{ppm})=6.54\left(\mathrm{~s}, 2 \mathrm{H}, \mathrm{NH}_{2}\right), 7.22(\mathrm{~s}, 1 \mathrm{H}$, ArH), 7.44 (d, $2 \mathrm{H}, J=8.4, \operatorname{ArH}), 7.52(\mathrm{~d}, 2 \mathrm{H}, J=8.8 \mathrm{~Hz}$, $\mathrm{ArH}), 7.73$ (d, $1 \mathrm{H}, J=15.6 \mathrm{~Hz},-\mathrm{C}=\mathrm{HC}-), 7.76$ (s, $1 \mathrm{H}, \mathrm{A}-$ $\mathrm{H}), 7.83(\mathrm{~d}, 1 \mathrm{H}, J=15.6 \mathrm{~Hz},-\mathrm{C}=\mathrm{HC}-), 7.91$ (d, $2 \mathrm{H}, J=$ 8.8, ArH), 7.7 (s, 1H, ArH), 7.80-7.85 (m, 3H, ArH), 8.01 (s, 1H, ArH), 10.11 (s, 1H, -NH), 10.41 (s, 1H, =N$\mathrm{NH}-\mathrm{C})$; LC-MS $M / Z: 461[\mathrm{M}+\mathrm{H}]^{+}$.

\section{2-((E)-3-(4-bromophenyl)-1-(4-((7-chloroquinolin-4-} yl)amino)phenyl)allylidene)hydrazinecarboxamide(7 g) Yield: $51 \%$; m.p.: $244-246{ }^{\circ} \mathrm{C}$; IR $\left(\mathrm{KBr} \mathrm{cm}^{-1}\right)$ : $3452\left(\mathrm{NH}_{2}\right.$, ), $1680(\mathrm{C}=\mathrm{O}), 1251$ (C-N); ${ }^{1} \mathrm{H}-\mathrm{NMR}$ (400 MHz, DMSO- $\left.d_{6}\right) \delta(\mathrm{ppm})=6.52\left(\mathrm{~s}, 2 \mathrm{H}, \mathrm{NH}_{2}\right), 7.16(\mathrm{~s}, 1 \mathrm{H}, \mathrm{ArH})$, 7.61(d, 2H, $J=8.4, A r H), 7.68(\mathrm{~d}, 2 \mathrm{H}, J=8.4 \mathrm{~Hz}, \mathrm{ArH})$, $7.74(\mathrm{~d}, 1 \mathrm{H}, J=15.6 \mathrm{~Hz},-\mathrm{C}=\mathrm{HC}-), 7.78(\mathrm{~s}, 1 \mathrm{H}, \mathrm{ArH})$, $7.81(\mathrm{~d}, 1 \mathrm{H}, J=15.6 \mathrm{~Hz},-\mathrm{C}=\mathrm{HC}-), 7.90(\mathrm{~d}, 2 \mathrm{H}, J=8.0$, ArH), 8.00 (s, 1H, ArH), 8.06 (s, 1H, ArH), 8.27 (d, 2H, $J=8.8 \mathrm{~Hz}, \mathrm{ArH}), 8.62(\mathrm{~s}, 1 \mathrm{H}, \mathrm{ArH}), 10.18(\mathrm{~s}, 1 \mathrm{H}, \mathrm{NH})$, 10.83 (s, 1H, =N-NH-C); LC-MS M/Z: $522[\mathrm{M}+\mathrm{H}]^{+}$.

\section{2-((E)-1-(4-((7-chloroquinolin-4-yl)amino)phenyl)-3-(4-} nitrophenyl)allylidene)hydrazinecarboxamide(7 $h$ )

Yield: $70 \%$; m.p.: $196-198^{\circ} \mathrm{C}$; IR $\left(\mathrm{KBr} \mathrm{cm}^{-1}\right)$ : 3398 $\left(\mathrm{NH}_{2}\right), 1675(\mathrm{C}=\mathrm{O}), 1585\left(\mathrm{NO}_{2}\right), 1371(\mathrm{C}-\mathrm{N}) ;{ }^{1} \mathrm{H}-\mathrm{NMR}$ $\left(400 \mathrm{MHz}, \mathrm{DMSO}-d_{6}\right) \delta(\mathrm{ppm})=6.50\left(\mathrm{~s}, 2 \mathrm{H}, \mathrm{NH}_{2}\right), 7.20$ (s, $1 \mathrm{H}, \operatorname{ArH}), 7.42$ (d, 2H, J=8.8 Hz, ArH), $7.62(\mathrm{~d}, 2 \mathrm{H}$, $J=8.8 \mathrm{~Hz}, \mathrm{ArH}$ ), 7.80 (d, $1 \mathrm{H}, J=15.6 \mathrm{~Hz},-\mathrm{C}=\mathrm{HC}-), 7.83$ (d, $1 \mathrm{H}, J=15.6 \mathrm{~Hz},-\mathrm{C}=\mathrm{HC}-), 7.9(\mathrm{~s}, 1 \mathrm{H}, \mathrm{ArH}), 8.02(\mathrm{~d}$, $2 \mathrm{H}, J=8.4, \mathrm{ArH}), 8.16$ (s, 1H, ArH), 8.29 (d, 2H, $J=8.4$ $\mathrm{Hz}, \mathrm{ArH}$ ), 8.53 (s, 1H, ArH), 8.62 (s, 1H, ArH), 10.30 (s, $1 \mathrm{H}, \mathrm{NH}), 10.76$ (s, 1H, =N-NH-C); LC-MS M/Z: 488 $[\mathrm{M}+\mathrm{H}]^{+}$.

2-((E)-1-(4-((7-chloroquinolin-4-yl)amino)phenyl)-3-(4hydroxyphenyl)allylidene)hydrazinecarboxamide(7i)

Yield: 45\%; m.p.: $240-242{ }^{\circ} \mathrm{C}$; IR $\left(\mathrm{KBr} \mathrm{cm} \mathrm{cm}^{-1}\right): 3360$ $\left(\mathrm{NH}_{2}\right), 1680(\mathrm{C}=\mathrm{O}), 1251(\mathrm{C}-\mathrm{N}) ;{ }^{1} \mathrm{H}-\mathrm{NMR}(400 \mathrm{MHz}$, DMSO-d $)_{6} \delta(\mathrm{ppm})=6.53\left(\mathrm{~s}, 2 \mathrm{H}, \mathrm{NH}_{2}\right), 7.23(\mathrm{~s}, 1 \mathrm{H}$, ArH), 7.56 (d, 2H, J=8.8 Hz, ArH), 7.68 (d, 2H, $J=8.8$ $\mathrm{Hz}, \mathrm{ArH}), 7.78$ (d, $1 \mathrm{H}, J=15.6 \mathrm{~Hz},-\mathrm{C}=\mathrm{HC}-), 7.81(\mathrm{~d}, 1 \mathrm{H}$, $J=15.6 \mathrm{~Hz},-\mathrm{C}=\mathrm{HC}-), 7.7$ (s, 1H, ArH), 8.0 (d, 2H, $J=$ 8.2, $\mathrm{ArH}), 8.16(\mathrm{~s}, 1 \mathrm{H}, \mathrm{ArH}), 8.26(\mathrm{~d}, 2 \mathrm{H}, J=8.4 \mathrm{~Hz}$, ArH), 8.51 (s, 1H, ArH), 8.60 (s, 1H, ArH), 9.89 (s, 1H, $\mathrm{OH}), 10.32$ (s, 1H, NH), 10.71 (s, 1H, =N-NH-C); LCMS $M / Z: 459[\mathrm{M}+\mathrm{H}]^{+}$.

\section{2-((E)-1-(3-((7-chloroquinolin-4-yl)amino)phenyl)-3-(p-} tolyl)allylidene)hydrazinecarbothioamide(8a)

Yield: $50 \%$; m.p.: $238-240{ }^{\circ} \mathrm{C}$; IR $\left(\mathrm{KBr} \mathrm{cm}^{-1}\right)$ : 3350 $\left(\mathrm{NH}_{2}\right), 1575(\mathrm{C}=\mathrm{C}), 1352(\mathrm{C}=\mathrm{S}), 1261(\mathrm{C}-\mathrm{N}) ;{ }^{1} \mathrm{H}-\mathrm{NMR}$ $\left(400 \mathrm{MHz}, \mathrm{DMSO}-d_{6}\right) \delta(\mathrm{ppm})=2.62\left(\mathrm{~s}, 3 \mathrm{H}, \mathrm{CH}_{3}\right), 6.99$ (s, 1H, ArH), 7.20-7.15 (m, 4H, ArH), 7.25 (d, 1H, J= $15.6 \mathrm{~Hz},-\mathrm{C}=\mathrm{HC}-), 7.37$ (d, 2H, J=8.4 Hz, ArH), 7.50 (d, $1 \mathrm{H}, J=15.6 \mathrm{~Hz},-\mathrm{C}=\mathrm{HC}-), 7.59$ (d, 2H, $J=8.4 \mathrm{~Hz}, \mathrm{ArH})$, 7.76 (s, 1H, ArH), 7.85 (s, 1H, NH $\mathrm{NH}_{2}, 7.88\left(\mathrm{~s}, 1 \mathrm{H}, \mathrm{NH}_{2}\right.$ ), 7.92 (s, 1H, ArH), 8.26 (s, 1H, ArH), 8.46 (s, 1H, ArH), 9.25 (s, 1H, NH), 11.25 (s, 1H, =N-NH-C); LC-MS M/Z: $473[\mathrm{M}+\mathrm{H}]^{+}$.

\section{2-((E)-1-(3-((7-chloroquinolin-4-yl)amino)phenyl)-3-(4-} Methoxyphenyl)allylidene)hydrazinecarbothioamide(8b) Yield: $43 \%$; m.p.: $210-212{ }^{\circ} \mathrm{C}$; IR $\left(\mathrm{KBr} \mathrm{cm}{ }^{-1}\right)$ : 3365 $\left(\mathrm{NH}_{2}\right), 1568(\mathrm{C}=\mathrm{C}), 1350(\mathrm{C}=\mathrm{S}), 1255\left(\mathrm{OCH}_{3}\right) ;{ }^{1} \mathrm{H}-\mathrm{NMR}$ $\left(400 \mathrm{MHz}, \mathrm{DMSO}-d_{6}\right) \delta(\mathrm{ppm})=3.81\left(\mathrm{~s}, 3 \mathrm{H}, \mathrm{OCH}_{3}\right)$, 7.42-7.35 (m, 4H, ArH), 7.67-7.58 (m, 3H, ArH, $-\mathrm{NH}_{2}$ ), $7.71\left(\mathrm{~s}, 1 \mathrm{H}, \mathrm{NH}_{2}\right), 7.72(\mathrm{~s}, 1 \mathrm{H}, \mathrm{ArH}), 7.73(\mathrm{~d}, 1 \mathrm{H}, J=$ 
$15.6 \mathrm{~Hz},-\mathrm{C}=\mathrm{HC}-), 7.76(\mathrm{~d}, 1 \mathrm{H}, J=15.6 \mathrm{~Hz},-\mathrm{C}=\mathrm{HC}-)$, 7.84 (d, 2H, $J=8.8 \mathrm{~Hz}, \operatorname{ArH}), 7.93$ (s, 1H, ArH), 8.04 (s, $1 \mathrm{H}, \mathrm{ArH}), 8.43$ (s, 1H, ArH), 8.50 (s, 1H, ArH), 9.26 (s, $1 \mathrm{H}, \mathrm{NH}), 11.75$ (s, 1H, =N-NH-C); LC-MS M/Z: 489 $[\mathrm{M}+\mathrm{H}]^{+}$.

\section{2-((E)-1-(3-((7-chloroquinolin-4-yl)amino)phenyl)-3-(3,4-} dimethoxyphenyl)allylidene)hydrazinecarbothioamide(8c)

Yield: $41 \%$; m.p.: $120-122^{\circ} \mathrm{C}$; IR $\left(\mathrm{KBr} \mathrm{cm}^{-1}\right): 3655$ $\left(\mathrm{NH}_{2}\right), 1516(\mathrm{C}=\mathrm{C}), 1313(\mathrm{C}=\mathrm{S}), 1267(\mathrm{C}-\mathrm{N}), 1560(\mathrm{C}=$ C), $1183\left(\mathrm{OCH}_{3}\right) ;{ }^{1} \mathrm{H}-\mathrm{NMR}\left(400 \mathrm{MHz}, \mathrm{DMSO}-d_{6}\right) \delta$ $(\mathrm{ppm})=3.81\left(\mathrm{~s}, 3 \mathrm{H}, \mathrm{OCH}_{3}\right), 3.84\left(\mathrm{~s}, 3 \mathrm{H},-\mathrm{OCH}_{3}\right), 7.39$ (s, 1H, ArH), $7.41(\mathrm{~s}, 1 \mathrm{H}, \mathrm{ArH}), 7.65\left(\mathrm{~s}, 1 \mathrm{H},-\mathrm{NH}_{2}\right)$, 7.70-7.66 (m, 3H, ArH), $7.73(\mathrm{~d}, 1 \mathrm{H}, J=15.6 \mathrm{~Hz},-\mathrm{C}=$ HC-), 7.80 (d, 1H, J=15.6 Hz, -C=HC-), 7.92-7.85 (m, $3 \mathrm{H}, \mathrm{ArH}), 7.94(\mathrm{~s}, 1 \mathrm{H}, \mathrm{ArH}), 8.01$ (s, 1H, ArH), 8.1 (s, $\left.1 \mathrm{H},-\mathrm{NH}_{2}\right), 8.14(\mathrm{~s}, 1 \mathrm{H}, \mathrm{ArH}), 8.49$ (s, 1H, ArH), 9.71 (s, $1 \mathrm{H}, \mathrm{NH}), 11.95$ (s, 1H, =N-NH-C); LC-MS $M / Z: 519$ $[\mathrm{M}+\mathrm{H}]^{+}$.

\section{2-((E)-1-(3-((7-chloroquinolin-4-yl)amino)phenyl)-3-(3,4,5-} trimethoxyphenyl)allylidene)hydrazinecarbothioamide(8d) Yield: $60 \%$; m.p.: $240-242{ }^{\circ} \mathrm{C}$; IR $\left(\mathrm{KBr} \mathrm{cm}^{-1}\right): 3352$ $\left(\mathrm{NH}_{2}\right), 1560(\mathrm{C}=\mathrm{C}), 1352(\mathrm{C}=\mathrm{S}), 1246(\mathrm{C}-\mathrm{N}), 1182$ $\left(\mathrm{OCH}_{3}\right) ;{ }^{1} \mathrm{H}-\mathrm{NMR}\left(400 \mathrm{MHz}, \mathrm{DMSO}-d_{6}\right) \delta(\mathrm{ppm})=3.70$ $\left(\mathrm{s}, 3 \mathrm{H}, \mathrm{OCH}_{3}\right), 3.84\left(\mathrm{~s}, 6 \mathrm{H}, \mathrm{OCH}_{3}\right), 7.22(\mathrm{~s}, 1 \mathrm{H}, \mathrm{ArH})$, 7.64-7.58 (m, 4H, ArH), 7.67 (s, 1H, ArH), 7.71(d, 1H, $J=15.6,-\mathrm{C}=\mathrm{HC}-), 7.84\left(\mathrm{~s}, 1 \mathrm{H}, \mathrm{NH}_{2}\right), 7.85(\mathrm{~d}, 1 \mathrm{H}, J=$ 15.6, -C=HC-), 7.91 (s, 1H, $\mathrm{NH}_{2}$ ), 7.97 (s 2H, ArH), 8.02 (s, 1H, ArH), 8.43 (s, 1H, ArH), 8.51 (s, 1H, ArH), 9.28 (s, 1H, NH), 10.96 (s, 1H, =N-NH-C); LC-MS M/Z: 549 $[\mathrm{M}+\mathrm{H}]^{+}$.

\section{2-((E)-3-(4-chlorophenyl)-1-(3-((7-chloroquinolin-4-} yl)amino)phenyl)allylidene)hydrazinecarbothioamide(8e) Yield: 66\%; m.p.: $212-214{ }^{\circ} \mathrm{C}$; IR $\left(\mathrm{KBr} \mathrm{cm}^{-1}\right)$ : 3338 $\left(\mathrm{NH}_{2}\right), 1597$ (C=C), 1350 (C=S), 1261 (C-N); ${ }^{1} \mathrm{H}-\mathrm{NMR}$ $\left(400 \mathrm{MHz}, \mathrm{DMSO}-d_{6}\right) \delta(\mathrm{ppm})=7.21(\mathrm{~s}, 1 \mathrm{H}, \mathrm{ArH}), 7.46$ (d, $1 \mathrm{H}, J=15.6 \mathrm{~Hz},-\mathrm{C}=\mathrm{HC}-), 7.52(\mathrm{~d}, 2 \mathrm{H}, J=8.4 \mathrm{~Hz}$, $\mathrm{ArH}), 7.59$ (d,1H, $J=15.6 \mathrm{~Hz},-\mathrm{C}=\mathrm{HC}-), 7.61$ (d, 2H, $J=$ 8.4 Hz, ArH), 7.79-7.63 (m, 4H, ArH), 7.81 (s, 1H, $\mathrm{NH}_{2}$ ), 7.85 (s, 1H, ArH), 7.93 (s, 1H, NH NH, 8.20 (s, 1H, ArH), 8.24 (s, 1H, ArH), 8.47(s, 1H, ArH), 9.29 (s, 1H, NH), 11.12 (s, 1H, =N-NH-C); LC-MS $M / Z: 494[\mathrm{M}+\mathrm{H}]^{+}$.

\section{2-((E)-1-(3-((7-chloroquinolin-4-yl)amino)phenyl)-3-(4-} fluorophenyl)allylidene)hydrazinecarbothioamide(8f)

Yield: $60 \%$; m.p.: $234-236{ }^{\circ} \mathrm{C}$; IR $\left(\mathrm{KBr} \mathrm{cm}^{-1}\right)$ : 3358 $\left(\mathrm{NH}_{2}\right), 1591$ (C=C), 1350 (C=S); ${ }^{1} \mathrm{H}-\mathrm{NMR}(400 \mathrm{MHz}$, DMSO- $\left.d_{6}\right) \delta(\mathrm{ppm})=7.21(\mathrm{~s}, 1 \mathrm{H}, \mathrm{ArH}), 7.41-7.34(\mathrm{~m}$, $4 \mathrm{H}, \mathrm{ArH}), 7.48$ (d,1H, J=15.6 Hz, -C=HC-), 7.57 (d, 2H, $J=8.4 \mathrm{~Hz}, \mathrm{ArH}), 7.63(\mathrm{~d}, 2 \mathrm{H}, J=8.4 \mathrm{~Hz}, \mathrm{ArH}), 7.76(\mathrm{~d}$, $1 \mathrm{H}, J=15.6 \mathrm{~Hz},-\mathrm{C}=\mathrm{HC}-), 7.88\left(\mathrm{~s}, 1 \mathrm{H}, \mathrm{NH}_{2}\right), 7.83(\mathrm{~s}, 1 \mathrm{H}$, $\mathrm{ArH}), 7.95$ (s, $\left.1 \mathrm{H}, \mathrm{NH}_{2}\right), 8.22(\mathrm{~s}, 1 \mathrm{H}, \mathrm{ArH}), 8.31(\mathrm{~s}, 1 \mathrm{H}$,
ArH), 8.45 (s, 1H, ArH), 9.25 (s, 1H, NH), 11.22 (s, 1H, =N-NH-C); LC-MS M/Z: $477[\mathrm{M}+\mathrm{H}]^{+}$.

2-((E)-3-(4-bromophenyl)-1-(3-((7-chloroquinolin-4yl)amino)phenyl)allylidene)hydrazinecarbothioamide(8 g) Yield: $55 \%$; m.p.: $238-240{ }^{\circ} \mathrm{C}$; IR $\left(\mathrm{KBr} \mathrm{cm}^{-1}\right)$ : 3364 $\left(\mathrm{NH}_{2}\right), 1575(\mathrm{C}=\mathrm{C}), 1355(\mathrm{C}=\mathrm{S}) ;{ }^{1} \mathrm{H}-\mathrm{NMR}(400 \mathrm{MHz}$, DMSO- $\left.d_{6}\right) \delta(\mathrm{ppm})=7.21(\mathrm{~s}, 1 \mathrm{H}, \mathrm{ArH}), 7.45-7.37(\mathrm{~m}$, $4 \mathrm{H}, \mathrm{ArH}), 7.48$ (d, $1 \mathrm{H}, J=15.6 \mathrm{~Hz},-\mathrm{C}=\mathrm{HC}-), 7.53(\mathrm{~d}, 2 \mathrm{H}$, $J=8.4 \mathrm{~Hz}, \mathrm{ArH}), 7.68(\mathrm{~d}, 2 \mathrm{H}, J=8.4 \mathrm{~Hz}, \mathrm{ArH}), 7.75(\mathrm{~d}$, $1 \mathrm{H}, J=15.6 \mathrm{~Hz},-\mathrm{C}=\mathrm{HC}-), 7.83\left(\mathrm{~s}, 1 \mathrm{H}, \mathrm{NH}_{2}\right), 7.91(\mathrm{~s}, 1 \mathrm{H}$, ArH), 7.96 (s, 1H, $\left.\mathrm{NH}_{2}\right), 8.14(\mathrm{~s}, 1 \mathrm{H}, \mathrm{ArH}), 8.23$ (s, $1 \mathrm{H}$, ArH), 8.46 (s, 1H, ArH), 9.23 (s, 1H, NH), 11.21 (s, 1H, =N-NH-C); LC-MS M/Z: $538[\mathrm{M}+\mathrm{H}]^{+}$.

\section{2-((E)-1-(3-((7-chloroquinolin-4-yl)amino)phenyl)-3-(4-} nitrophenyl)allylidene)hydrazinecarbothioamide(8 $\mathrm{h}$ )

Yield: $47 \%$; m.p.: $218-220^{\circ} \mathrm{C}$; IR $\left(\mathrm{KBr} \mathrm{cm}^{-1}\right)$ : 3356 $\left(\mathrm{NH}_{2}\right), 1593\left(\mathrm{NO}_{2}\right), 1550(\mathrm{C}=\mathrm{C}), 1350(\mathrm{C}=\mathrm{S}) ;{ }^{1} \mathrm{H}-\mathrm{NMR}$ $\left(400 \mathrm{MHz}, \mathrm{DMSO}-d_{6}\right) \delta(\mathrm{ppm})=7.20(\mathrm{~s}, 1 \mathrm{H}, \mathrm{ArH})$, $7.52-7.43(\mathrm{~m}, 4 \mathrm{H}, \mathrm{ArH}), 7.56(\mathrm{~d}, 2 \mathrm{H}, J=8.4 \mathrm{~Hz}, \mathrm{ArH})$, $7.61(\mathrm{~d}, 1 \mathrm{H}, J=15.6 \mathrm{~Hz},-\mathrm{C}=\mathrm{HC}-), 7.64(\mathrm{~d}, 2 \mathrm{H}, J=7.6 \mathrm{~Hz}$, $\mathrm{ArH}), 7.69(\mathrm{~d}, 1 \mathrm{H}, J=15.6 \mathrm{~Hz},-\mathrm{C}=\mathrm{HC}-), 7.72(\mathrm{~s}, 1 \mathrm{H}$, $\left.\mathrm{NH}_{2}\right), 7.96\left(\mathrm{~s}, 1 \mathrm{H}, \mathrm{NH}_{2}\right), 8.16(\mathrm{~s}, 1 \mathrm{H}, \mathrm{ArH}), 8.31$ (s, $1 \mathrm{H}$, ArH), 8.36 (s, 1H, ArH), 8.48 (s, 1H, ArH), 9.68 (s, 1H, $\mathrm{NH}), 11.23$ (s, 1H, =N-NH-C); LC-MS $M / Z: 504[\mathrm{M}+$ $\mathrm{H}]^{+}$.

\section{2-((E)-1-(3-((7-chloroquinolin-4-yl)amino)phenyl)-3-(4-} hydroxyphenyl)allylidene)hydrazinecarbothioamide(8i) Yield: 57\%; m.p.: $250-242{ }^{\circ} \mathrm{C}$; IR $\left(\mathrm{KBr} \mathrm{cm}^{-1}\right): 3478(\mathrm{OH})$, $3360\left(\mathrm{NH}_{2}\right), 1590(\mathrm{C}=\mathrm{C}), 1361(\mathrm{C}=\mathrm{S}), 1265(\mathrm{C}-\mathrm{N}) ;{ }^{1} \mathrm{H}-$ NMR $\left(400 \mathrm{MHz}, \mathrm{DMSO}-d_{6}\right) \delta(\mathrm{ppm})=7.2(\mathrm{~s}, 1 \mathrm{H}, \mathrm{ArH})$, $7.20-7.15(\mathrm{~m}, 4 \mathrm{H}, \mathrm{ArH}), 7.25(\mathrm{~d}, 1 \mathrm{H}, J=15.6 \mathrm{~Hz},-\mathrm{C}=$ HC-), 7.38 (d, 2H, J=8.4 Hz, ArH), 7.50 (d, 1H, $J=15.6$ $\mathrm{Hz},-\mathrm{C}=\mathrm{HC}-$ ), 7.61 (d, 2H, J=8.4 Hz, ArH), 7.76 (s, 1H, $\mathrm{ArH}), 7.87$ (s, 1H, $\left.\mathrm{NH}_{2}\right), 7.93\left(\mathrm{~s}, 1 \mathrm{H}, \mathrm{NH}_{2}\right), 7.97(\mathrm{~s}, 1 \mathrm{H}$, ArH), 8.31 (s, 1H, ArH), 8.43 (s, 1H, ArH), 9.35 (s, 1H, $\mathrm{NH}), 9.98$ (s, 1H, OH), 11.22 (s, 1H, =N-NH-C); LC-MS $M / Z: 475[\mathrm{M}+\mathrm{H}]^{+}$.

\section{2-((E)-1-(3-((7-chloroquinolin-4-yl)amino)phenyl)-3-(p- tolyl)allylidene)hydrazinecarboxamide(9a)}

Yield: $55 \%$; m.p.: $192-194{ }^{\circ} \mathrm{C}$; IR $\left(\mathrm{KBr} \mathrm{cm}{ }^{-1}\right): 3365$ $\left(\mathrm{NH}_{2}\right), 1690(\mathrm{C}=\mathrm{O}), 1575(\mathrm{C}=\mathrm{C}), 1261(\mathrm{C}-\mathrm{N}) ;{ }^{1} \mathrm{H}-\mathrm{NMR}$ $\left(400 \mathrm{MHz}, \mathrm{DMSO}-d_{6}\right) \delta(\mathrm{ppm})=2.61\left(\mathrm{~s}, 3 \mathrm{H}, \mathrm{CH}_{3}\right), 6.73$ (s, 2H, $\mathrm{NH}_{2}$ ), 7.16 (s, 1H, ArH), 7.33-7.21 (m, 4H, ArH), $7.36(\mathrm{~d}, 1 \mathrm{H}, J=15.6 \mathrm{~Hz},-\mathrm{C}=\mathrm{HC}-), 7.41(\mathrm{~d}, 2 \mathrm{H}, J=8.4 \mathrm{~Hz}$, $\mathrm{ArH}), 7.53$ (d, 1H, $J=15.6 \mathrm{~Hz},-\mathrm{C}=\mathrm{HC}-), 7.61$ (d, 2H, $J=$ 8.4 Hz, ArH), 7.82 (s, 1H, ArH), 7.91 (s, 1H, ArH), 8.32 (s, 1H, ArH), 8.41 (s, 1H, ArH), 9.33 (s, 1H, NH), 10.93 (s, 1H, =N-NH-C); LC-MS M/Z: $457[\mathrm{M}+\mathrm{H}]^{+}$. 
2-((E)-1-(3-((7-chloroquinolin-4-yl)amino)phenyl)-3-(4methoxyphenyl) allylidene)hydrazinecarboxamide(9b) Yield: $40 \%$; m.p.: $188-190{ }^{\circ} \mathrm{C}$; IR $\left(\mathrm{KBr} \mathrm{cm} \mathrm{cm}^{-1}\right): 3365$ $\left(\mathrm{NH}_{2}\right), 1685(\mathrm{C}=\mathrm{O}), 1590(\mathrm{C}=\mathrm{C}), 1235\left(\mathrm{OCH}_{3}\right) ;{ }^{1} \mathrm{H}-$ NMR $\left(400 \mathrm{MHz}, \mathrm{DMSO}-d_{6}\right) \quad \delta(\mathrm{ppm})=3.83 \quad(\mathrm{~s}, 3 \mathrm{H}$, $\mathrm{OCH}_{3}$ ), 6.69 (s, 2H, NH$)_{2}$ ) 7.52-7.43 (m, 4H, ArH), 7.65 (d, 2H, ArH), 7.68 (s, 1H, ArH), $7.71(\mathrm{~d}, 1 \mathrm{H}, J=15.6 \mathrm{~Hz}$, -C=HC-), 7.75 (d, 1H, J=15.6 Hz, -C=HC-), 7.81 (d, 2H, $J=8.8 \mathrm{~Hz}, \operatorname{ArH}), 7.93$ (s, 1H, ArH), 8.01 (s, 1H, ArH), 8.42 (s, 1H, ArH), 8.47 (s, 1H, ArH), 9.34 (s, 1H, NH), 11.10 (s, 1H, =N-NH-C); LC-MS $M / Z: 473[\mathrm{M}+\mathrm{H}]^{+}$.

2-((E)-1-(3-((7-chloroquinolin-4-yl)amino)phenyl)-3-(3,4dimethoxyphenyl)allylidene)hydrazinecarboxamide(9c)

Yield: 51\%; m.p.: $254-256^{\circ} \mathrm{C}$; IR $\left(\mathrm{KBr} \mathrm{cm}^{-1}\right)$ : 3648 $\left(\mathrm{NH}_{2}\right), 1680(\mathrm{C}=\mathrm{O}), 1580(\mathrm{C}=\mathrm{C}), 1215\left(\mathrm{OCH}_{3}\right) ;{ }^{1} \mathrm{H}-$ NMR $\left(400 \mathrm{MHz}, \mathrm{DMSO}-d_{6}\right) \delta(\mathrm{ppm})=3.86 \quad(\mathrm{~s}, 3 \mathrm{H}$, $\left.\mathrm{OCH}_{3}\right), 3.89\left(\mathrm{~s}, 3 \mathrm{H},-\mathrm{OCH}_{3}\right), 6.69\left(\mathrm{~s}, 2 \mathrm{H},-\mathrm{NH}_{2}\right), 7.53(\mathrm{~s}$, $1 \mathrm{H}, \mathrm{ArH}), 7.68-7.59$ (m, 3H, ArH), 7.71 (d, 1H, J=15.6 $\mathrm{Hz},-\mathrm{C}=\mathrm{HC}-$ ) 7.79 (d, 1H, J=15.6 Hz, -C=HC-), 7.917.83 (m, 3H, ArH), 7.94 (s, 1H, ArH), 8.10 (s, 1H, ArH), 8.23 (s, 1H, ArH), 8.49 (s, 1H, ArH), 8.51(s, H, ArH), 9.36 (s, 1H, NH), 11.13 (s, 1H, =N-NH-C); LC-MS $M / Z$ : $503[\mathrm{M}+\mathrm{H}]^{+}$.

2-((E)-1-(3-((7-chloroquinolin-4-yl)amino)phenyl)-3-(3,4,5trimethoxyphenyl) allylidene)hydrazinecarboxamide(9d)

Yield: $60 \%$; m.p.: $258-260{ }^{\circ} \mathrm{C}$; IR $\left(\mathrm{KBr} \mathrm{cm}^{-1}\right)$ : 3363 $\left(\mathrm{NH}_{2}\right), 1690(\mathrm{C}=\mathrm{O}), 1565(\mathrm{C}=\mathrm{C}), 1248(\mathrm{C}-\mathrm{N}), 1189$ $\left(\mathrm{OCH}_{3}\right) ;{ }^{1} \mathrm{H}-\mathrm{NMR}\left(400 \mathrm{MHz}, \mathrm{DMSO}-d_{6}\right) \delta(\mathrm{ppm})=3.76$ $\left(\mathrm{s}, 3 \mathrm{H}, \mathrm{OCH}_{3}\right), 3.85\left(\mathrm{~s}, 6 \mathrm{H},-\mathrm{OCH}_{3}\right), 6.79\left(\mathrm{~s}, 2 \mathrm{H}, \mathrm{NH}_{2}\right)$, 7.41 (s, 1H, ArH), 7.81-7.7 (m, 4H, ArH), 7.92 (s, 1H, $\mathrm{ArH}), 7.83$ (d, 1H, J=15.6, -C=HC-), 7.87 (d, 1H, J= 15.6, -C=HC-), 7.96 (s 2H, ArH), 8.13 (s, 1H, ArH), 8.41 (s, 1H, ArH), 8.48 (s, 1H, ArH), 9.33 (s, 1H, NH), 10.83 (s, $1 \mathrm{H},=\mathrm{N}-\mathrm{NH}-\mathrm{C})$; LC-MS $M / Z: 533[\mathrm{M}+\mathrm{H}]^{+}$.

2-((E)-3-(4-chlorophenyl)-1-(3-((7-chloroquinolin-4yl)amino)phenyl)allylidene)hydrazinecarboxamide(9e)

Yield: $50 \%$; m.p.: $226-228^{\circ} \mathrm{C}$; IR $\left(\mathrm{KBr} \mathrm{cm}^{-1}\right)$ : 3354 $\left(\mathrm{NH}_{2}\right), 1686(\mathrm{C}=\mathrm{O}), 1583(\mathrm{C}=\mathrm{C}), 1270(\mathrm{C}-\mathrm{N}) ;{ }^{1} \mathrm{H}-\mathrm{NMR}$ $\left(400 \mathrm{MHz}, \mathrm{DMSO}-d_{6}\right) \delta(\mathrm{ppm})=6.73\left(\mathrm{~s}, 2 \mathrm{H}, \mathrm{NH}_{2}\right), 7.13$ (s, 1H, ArH), 7.48 (d, 1H, J=15.6 Hz, -C=HC-), 7.53 (d, $2 \mathrm{H}, J=8.4 \mathrm{~Hz}, \mathrm{ArH}), 7.58(\mathrm{~d}, 1 \mathrm{H}, J=15.6 \mathrm{~Hz},-\mathrm{C}=\mathrm{HC}-)$, $7.65(\mathrm{~d}, 2 \mathrm{H}, J=8.4 \mathrm{~Hz}, \mathrm{ArH}), 7.81-7.75(\mathrm{~m}, 4 \mathrm{H}, \mathrm{ArH})$, 7.83 (s, 1H, ArH), 8.22 (s, 1H, ArH), 8.35 (s, 1H, ArH), 8.43 (s, 1H, ArH), 9.31 (s, 1H, NH), $10.86(\mathrm{~s}, 1 \mathrm{H},=\mathrm{N}$ $\mathrm{NH}-\mathrm{C})$; LC-MS $M / Z: 477[\mathrm{M}+\mathrm{H}]^{+}$.

\section{2-((E)-1-(3-((7-chloroquinolin-4-yl)amino)phenyl)-3-(4-} fluorophenyl)allylidene)hydrazinecarboxamide(9f)

Yield: $55 \%$; m.p.: $198-200{ }^{\circ} \mathrm{C}$; IR $\left(\mathrm{KBr} \mathrm{cm}^{-1}\right)$ : 3360 $\left(\mathrm{NH}_{2}\right), 1685(\mathrm{C}=\mathrm{O}), 1595(\mathrm{C}=\mathrm{C})$; ${ }^{1} \mathrm{H}-\mathrm{NMR}(400 \mathrm{MHz}$, DMSO- $\left.d_{6}\right) \delta(\mathrm{ppm})=6.73\left(\mathrm{~s}, 2 \mathrm{H}, \mathrm{NH}_{2}\right), 7.28(\mathrm{~s}, 1 \mathrm{H}$,
ArH), 7.45-7.36 (m, 4H, ArH), $7.49(\mathrm{~d}, 1 \mathrm{H}, J=15.6 \mathrm{~Hz}$, -C=HC-), 7.53 (d, 2H, J=8.4 Hz, ArH), 7.59 (d, 2H, J= $8.4 \mathrm{~Hz}, \mathrm{ArH}), 7.75$ (d, 1H, J=15.6 Hz, -C=HC-), 7.95 (s, $1 \mathrm{H}, \mathrm{ArH}), 8.26$ (s, 1H, ArH), 8.35 (s, 1H, ArH), 8.46 (s, $1 \mathrm{H}, \mathrm{ArH}), 9.29$ (s, 1H, NH), 10.98 (s, 1H, =N-NH-C); LC-MS M/Z: $461[\mathrm{M}+\mathrm{H}]^{+}$.

\section{2-((E)-3-(4-bromophenyl)-1-(3-((7-chloroquinolin-4- \\ yl)amino)phenyl)allylidene)hydrazinecarboxamide $(9 \mathrm{~g}$ )}

Yield: $60 \%$; m.p.: $306-308^{\circ} \mathrm{C}$; IR $\left(\mathrm{KBr} \mathrm{cm} \mathrm{cm}^{-1}\right): 3360$ $\left(\mathrm{NH}_{2}\right), 1686(\mathrm{C}=\mathrm{O}), 1570(\mathrm{C}=\mathrm{C}) ;{ }^{1} \mathrm{H}-\mathrm{NMR}(400 \mathrm{MHz}$, DMSO-d $) \delta(\mathrm{ppm})=6.75\left(\mathrm{~s}, 2 \mathrm{H}, \mathrm{NH}_{2}\right), 7.14(\mathrm{~s}, 1 \mathrm{H}$, ArH), 7.49-7.38 (m, 4H, ArH), 7.52 (d, $1 \mathrm{H}, J=15.6 \mathrm{~Hz}$, -C=HC-), 7.63 (d, 2H, J=8.4 Hz, ArH), 7.71 (d, 2H, J= 8.4 Hz, ArH), 7.79 (d, 1H, J=15.6 Hz, -C=HC-), 7.93 (s, $1 \mathrm{H}, \mathrm{ArH}), 8.22$ (s, 1H, ArH), 8.29 (s, 1H, ArH), 8.45 (s, $1 \mathrm{H}, \mathrm{ArH}), 9.27$ (s, 1H, NH), 10.96 (s, 1H, =N-NH-C); LC-MS M/Z: $522[\mathrm{M}+\mathrm{H}]^{+}$.

\section{2-((E)-1-(3-((7-chloroquinolin-4-yl)amino)phenyl)-3-(4-} nitrophenyl)allylidene)hydrazinecarboxamide(9 $h$ )

Yield: $43 \%$; m.p.: $246-248{ }^{\circ} \mathrm{C}$; IR $\left(\mathrm{KBr} \mathrm{cm}{ }^{-1}\right): 3360$ $\left(\mathrm{NH}_{2}\right), 1690(\mathrm{C}=\mathrm{O}), 1595\left(\mathrm{NO}_{2}\right), 1565(\mathrm{C}=\mathrm{C}) ;{ }^{1} \mathrm{H}-\mathrm{NMR}$ $\left(400 \mathrm{MHz}, \mathrm{DMSO}-d_{6}\right) \delta(\mathrm{ppm})=6.72\left(\mathrm{~s}, 2 \mathrm{H}, \mathrm{NH}_{2}\right), 7.20$ (s, 1H, ArH), 7.53-7.48 (m, 4H, ArH), 7.56 (d 2H, J= $8.4 \mathrm{~Hz}, \mathrm{ArH}), 7.65$ (d, 2H, J=7.6 Hz, ArH), $7.73(\mathrm{~d}, 1 \mathrm{H}$, $J=15.6 \mathrm{~Hz},-\mathrm{C}=\mathrm{HC}-), 7.96(\mathrm{~d}, 1 \mathrm{H}, J=15.6 \mathrm{~Hz},-\mathrm{C}=\mathrm{HC}-)$, 8.13 (s, 1H, ArH), 8.26 (s, 1H, ArH), 8.33 (s, 1H, ArH), 8.47 (s, 1H, ArH), 9.36 (s, 1H, NH), 10.87 (s, 1H, =N$\mathrm{NH}-\mathrm{C})$; LC-MS $M / Z: 488[\mathrm{M}+\mathrm{H}]^{+}$.

\section{2-((E)-1-(3-((7-chloroquinolin-4-yl)amino)phenyl)-3-(4-} hydroxyphenyl)allylidene)hydrazinecarboxamide(9i)

Yield: 43\%; m.p.: $234-236^{\circ} \mathrm{C}$; IR $\left(\mathrm{KBr} \mathrm{cm}^{-1}\right): 3362$ $\left(\mathrm{NH}_{2}\right), 1685$ (C=O), $1593(\mathrm{C}=\mathrm{C}), 1265(\mathrm{C}-\mathrm{N}) ;{ }^{1} \mathrm{H}-\mathrm{NMR}$ $\left(400 \mathrm{MHz}, \mathrm{DMSO}-d_{6}\right) \delta(\mathrm{ppm})=6.67\left(\mathrm{~s}, 2 \mathrm{H}, \mathrm{NH}_{2}\right), 7.10$ (s, 1H, ArH), 7.23-7.14 (m, 4H, ArH), 7.27 (d, 1H, J= $15.6 \mathrm{~Hz},-\mathrm{C}=\mathrm{HC}-), 7.49$ (d, 2H, J=8.4 Hz, ArH), 7.62 (d, $1 \mathrm{H}, J=15.6 \mathrm{~Hz},-\mathrm{C}=\mathrm{HC}-), 7.73(\mathrm{~d}, 2 \mathrm{H}, J=8.4 \mathrm{~Hz}, \mathrm{ArH})$, 7.98 (s, 1H, ArH), 8.15 (s, 1H, ArH), 8.36 (s, 1H, ArH), 8.46 (s, 1H, ArH), 9.29 (s, 1H, NH), 9.85 (s, 1H, OH), $10.93(\mathrm{~s}, 1 \mathrm{H},=\mathrm{N}-\mathrm{NH}-\mathrm{C})$; LC-MS $M / Z: 459[\mathrm{M}+\mathrm{H}]^{+}$.

\section{Discussions}

\section{Chemistry}

The reaction sequence employed for synthesis of the title compounds (6a-i), (7a-i), (8a-i), and (9a-i) are illustrated in Scheme 1. Initially, compounds 1-(4-((7-chloroquinolin-4-yl) amino)phenyl)ethanone (2) and 1-(3-((7chloroquinolin-4-yl)amino) phenyl)ethanone (3) were prepared with slight modification on the basis of the previously reported methods [21]. Their IR spectra showed the appearance of characteristic band at around $3564-3651 \mathrm{~cm}^{-1}$ attributed to amino $(\mathrm{NH})$ group and 


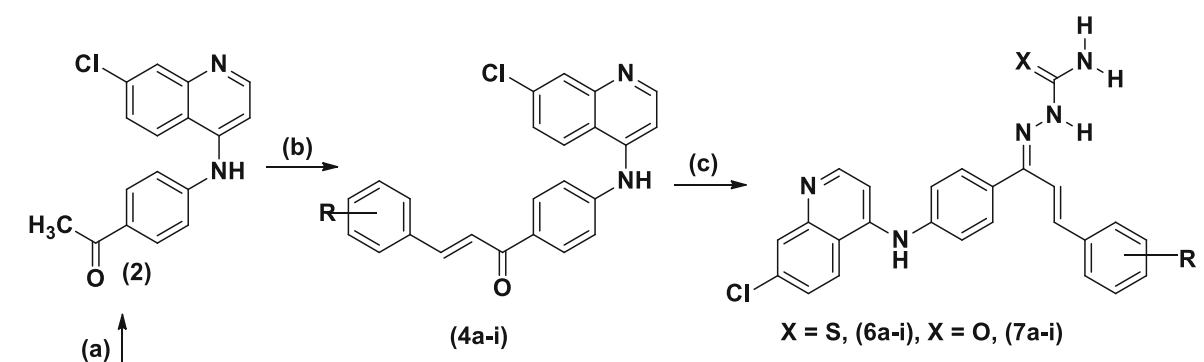<smiles>Clc1ccc2c(Cl)ccnc2c1</smiles><smiles>CC(=O)c1cccc(Nc2ccnc3cc(Cl)ccc23)c1</smiles>

(3)

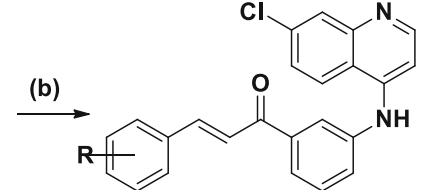

(5a-i)

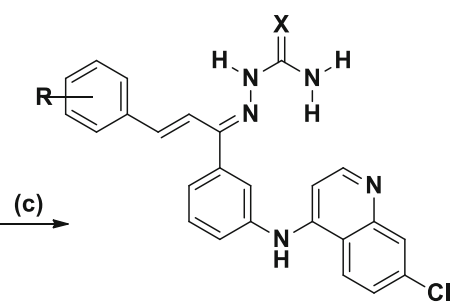

$X=S,(8 a-i), X=0,(9 a-i)$

Scheme 1 Reagents and conditions: (a) dry methanol, reflux 9-10 h; (b) methanol, NaOH, THF, RT; (c) thiosemicarbazide or semicarbazide hydrochloride, dry isopropyl alcohol, and glacial acetic acid, reflux $36 \mathrm{~h}$

carbonyl $(\mathrm{C}=\mathrm{O})$ stretching frequency at around 1684 $\mathrm{cm}^{-1}$, while their ${ }^{1} \mathrm{H}$ NMR spectrum indicated singlet signal at around $\delta 11.16$ and $11.33 \mathrm{ppm}$ for amine $(\mathrm{NH})$ protons. Further, these compounds were confirmed by $\mathrm{D}_{2} \mathrm{O}$ exchange experiment. The key intermediate $\alpha, \beta$ unsaturated ketones (chalcones) $(\mathbf{4} \mathbf{a}-\mathbf{i})$ and $(\mathbf{5 a}-\mathbf{i})$ were synthesized by reacting 1-(4-((7-chloroquinolin-4-yl)amino)phenyl)ethanone (2) or 1-(3-((7-chloroquinolin-4yl)amino)phenyl)ethanone (3) with appropriate aldehydes by conventional base-catalyzed Claisen-Schmidt condensation. The IR spectra of the compounds (4a-i) and $(\mathbf{5 a}-\mathbf{i})$ showed new band at $1530 \mathrm{~cm}^{-1}$ attributed to an alkene $(C=C)$ group of $\alpha, \beta$-unsaturated ketones. In addition, two sets of protons with a trans coupling constant $(J=16 \mathrm{~Hz})$ were present at $\delta 7.72$ and $8.05 \mathrm{ppm}$ which confirms the completion of Claisen-Schmidt condensation reactions. Reaction of chalcones $(\mathbf{4 a - i})$ and (5a-i) with thiosemicarbazide and semicarbazide hydrochloride in dry isopropyl alcohol and the presence of glacial acetic acid for $36 \mathrm{~h}$ afforded the target thiosemicarbazone (6a-i) and (8a-i) and semicarbazone $(7 \mathbf{a}-\mathbf{i})$ and $(\mathbf{9 a - i})$ derivatives respectively.

The structures of target compounds were established by using different spectroscopic techniques, including FTIR, NMR, and mass spectroscopy. The FTIR spectra of these compounds showed a medium to strong band for $\mathrm{C}=\mathrm{N}$ stretch which appeared in the range 1620 to $1560 \mathrm{~cm}^{-1}, \mathrm{C}=\mathrm{S}$ absorption band appeared at 1341 to $1356 \mathrm{~cm}^{-1}$, and semicarbazone derivatives showed $\mathrm{C}=\mathrm{O}$ absorption band at 1661 to $1690 \mathrm{~cm}^{-1}$. In addition, FTIR spectra showed the appearance of characteristic bands at 3350 and $3290 \mathrm{~cm}^{-1}$ assignable for amino (NH) group, while their proton NMR spectrum indicated singlet at around $\delta 11.69-11.82 \mathrm{ppm}$ for $=\mathrm{N}-\mathrm{NH}-\mathrm{C}$ - proton. The two amino $\left(-\mathrm{NH}_{2}\right)$ protons appeared as two singlets in the range at 7.67 and $8.2 \mathrm{ppm}$. Finally, formation of these compounds was also evidenced by mass spectral data.

\section{Biological activities \\ Antitubercular activity}

The new thiosemicarbazone derivatives $(\mathbf{6 a}-\mathbf{i})$ and $(\mathbf{8 a}-\mathbf{i})$ and semicarbazide compounds $(7 \mathbf{a}-\mathbf{i})$ and $(9 \mathbf{a}-\mathbf{i})$ were evaluated against Mycobacterium tuberculosis (MTB) $\mathrm{H}_{37} \mathrm{Rv}$ (ATCC 27294) by using the microplate alamar blue assay (MABA) [22, 23]. The minimum inhibitory concentration (MIC) was determined for each derivative which was measured as the minimum concentration of the compound required to completely inhibit the bacterial growth. Isoniazid, pyrazinamide, and ciprofloxacin were used as standard drugs for comparison. Antitubercular activity results are summarized in Table 1 . From the results, it was observed that among the tested compounds, the most active compounds $7 \mathbf{c}$ and $7 \mathbf{g}$ exhibited Mycobacterium tuberculosis inhibition at $1.56 \mu \mathrm{M}$ as compared to standard isoniazid $(\mathrm{MIC}=1.56 \mu \mathrm{M})$. Three compounds $7 \mathbf{a}, 7 \mathbf{i}$, and $9 \mathbf{a}$ inhibited MTB with MIC value of $6.25 \mu \mathrm{M}$, when compared to ciprofloxacin $(\mathrm{MIC}=12.5 \mu \mathrm{M})$ and pyrazinamide $(\mathrm{MIC}=50 \mu \mathrm{M})$. Nine compounds $6 \mathbf{6}, \mathbf{6 b}, \mathbf{6 d}, \mathbf{6 e}, 7 \mathrm{~d}, 7 \mathrm{e}, \mathbf{8 a}, \mathbf{8 b}, \mathbf{9 b}$, and $9 \mathrm{c}$ are 
Table 1 Physical data and antimycobacterial activities of compound (6a-i), (7a-i), (8a-i), and (9a-i) against MTB $\mathrm{H}_{37} \mathrm{Rv}$

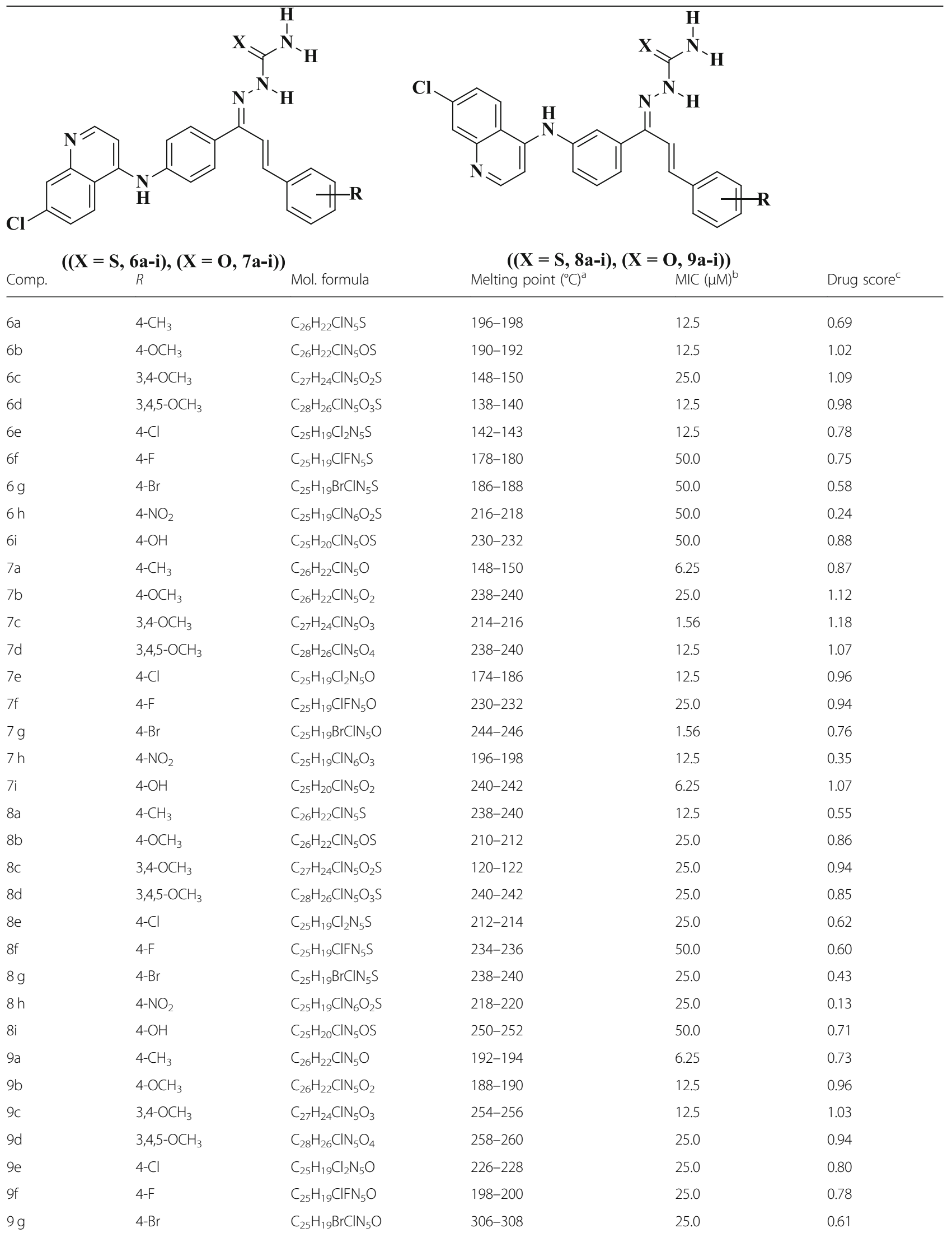


Table 1 Physical data and antimycobacterial activities of compound (6a-i), (7a-i), (8a-i), and (9a-i) against MTB $\mathrm{H}_{37} \mathrm{Rv}($ Continued)

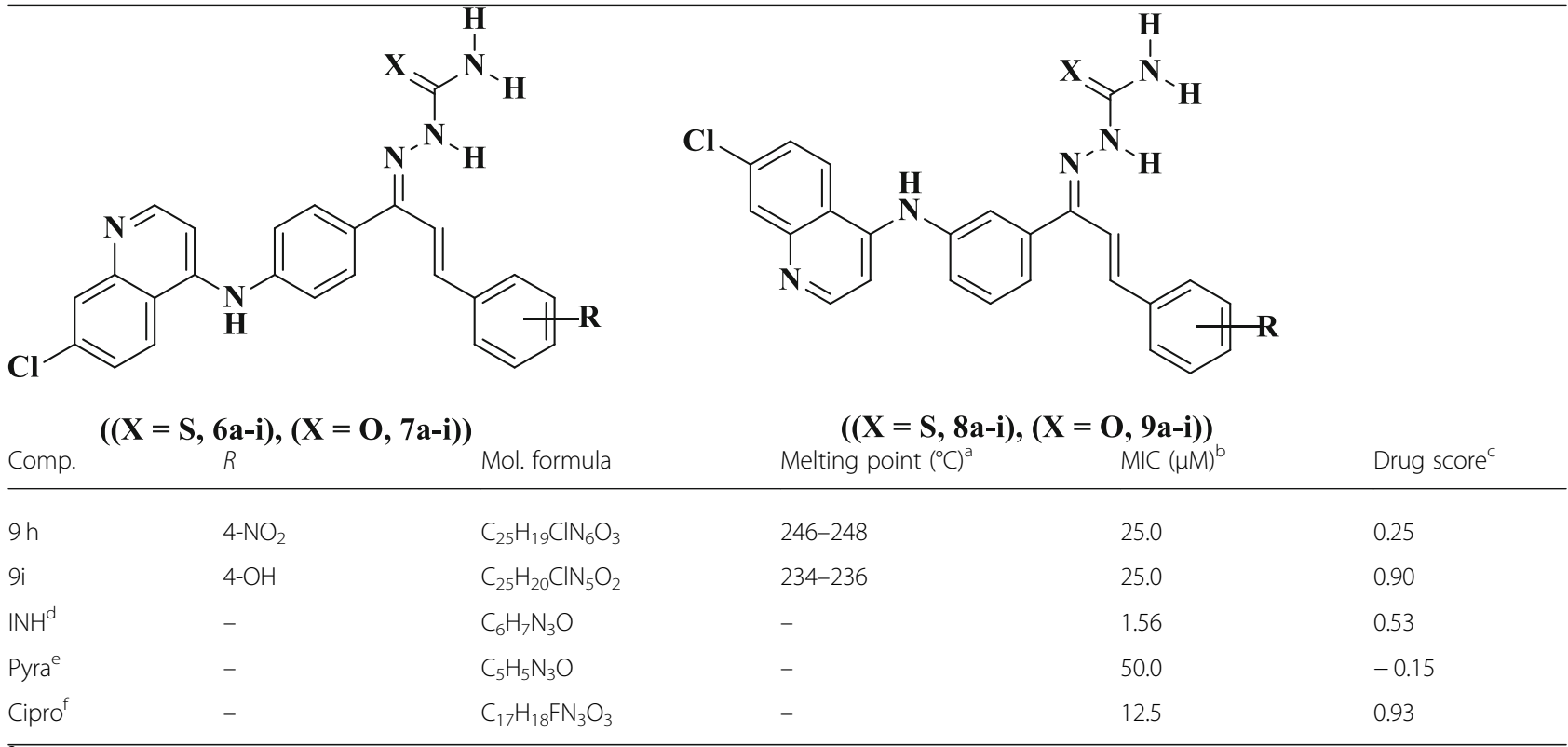

${ }^{a}$ The melting points were determined in open capillary tubes and are uncorrected

${ }^{\mathrm{b}}$ Minimum inhibitory concentration against Mycobacterium tuberculosis $\mathrm{H}_{37} \mathrm{Rv}$

${ }^{c}$ Calculated using molsoft (http://molsoft.com/mprop/)

dsoniazid

epyrazinamide

${ }^{f}$ Ciprofloxacin

found to be equally active as ciprofloxacin $(12.5 \mu \mathrm{M})$. When compared to pyrazinamide $(50.0 \mu \mathrm{M})$, most of the compounds were found to be more active, though 34 compounds were less potent than the first-line antitubercular drug isoniazid. Further, the following features of structure-activity relationship (SAR) of 7 -chloro- $\mathrm{N}$ phenylquinolin-4-amine derivatives $(\mathbf{6 a}-\mathbf{i}),(7 \mathbf{a}-\mathbf{i}),(\mathbf{8} \mathbf{a}-\mathbf{i})$, and (9a-i) have been drawn against the inhibition of Mycobacterium tuberculosis. The thiosemicarbazide (6ai) compounds with electron donating groups like $4-\mathrm{CH}_{3}$ (6a), $4-\mathrm{OCH}_{3}(\mathbf{6 b})$, and $3,4,5-\mathrm{OCH}_{3}(\mathbf{6 d})$ have shown activity at $12.5 \mu \mathrm{M}$. When the same methoxy group was substituted at $\mathrm{C}-3$ and $\mathrm{C}-4$ positions $\left(3,4-\mathrm{OCH}_{3}\right)$, the activity decreased to $25 \mu \mathrm{M}$ as shown by compound $\mathbf{6 c}$. Substitution of the C-4 position by a hydroxyl group (6i) further decreased the activity to $50 \mu \mathrm{M}$. Compounds $\mathbf{6 f}$, $\mathbf{6 g}$, and $\mathbf{6 h}$ with electron withdrawing groups like $\mathrm{F}, \mathrm{Br}$, and $\mathrm{NO}_{2}$ substituted at the $\mathrm{C}-4$ position showed very weak activity $(50 \mu \mathrm{M})$. However, the 4-chloro substituted compound 6e showed moderate activity $(12.5 \mu \mathrm{M})$.

Among the semicarbazones (6a-i), compounds $7 \mathbf{c}$ and $7 \mathrm{~g}$ displayed very promising activity at $1.56 \mu \mathrm{M}$, which was higher than the activities shown by their thiosemicarbazone counterparts. Compound $7 \mathbf{a}$ with an alkyl substituent $\left(4-\mathrm{CH}_{3}\right)$ showed better activity at $6.25 \mu \mathrm{M}$ as compared to $\mathbf{6 a}$, whereas compound $\mathbf{7 b}$ with an alkoxy $\left(4-\mathrm{OCH}_{3}\right)$ substituent displayed decreased activity as compared to $\mathbf{6 b}$. Compounds with 3,4,5- $\left(\mathrm{OCH}_{3}\right)_{3}$ group (7d) and 4-Cl group (7e) showed activities at $12.5 \mu \mathrm{M}$, similar to $\mathbf{6 d}$ and $\mathbf{6 e}$. Compound $7 \mathbf{g}$ with an electronegative group at position $\mathrm{C}-4$ proved to be the next most potent molecule by exhibiting inhibitory activity at $1.56 \mu \mathrm{M}$. Compounds $7 \mathbf{h}\left(4-\mathrm{NO}_{2}\right)$ and $7 \mathbf{i}(4-\mathrm{OH})$ displayed better inhibitory activities $(12.5 \mu \mathrm{M}$ and $6.25 \mu \mathrm{M})$ as compared to compounds $\mathbf{6 h}$ and $\mathbf{6 i}(50 \mu \mathrm{M})$. In the next series, the compound $\mathbf{8 a}$ with an electron donating 4- $\mathrm{CH}_{3}$ group has shown inhibitory activity at $12.5 \mu \mathrm{M}$. Other compounds $\mathbf{8 b}, \mathbf{8 c}$, and $\mathbf{8 d}$ with electron donating groups like 4- $\mathrm{OCH}_{3}, 3,4-\left(\mathrm{OCH}_{3}\right)_{2}$, and $3,4,5-\left(\mathrm{OCH}_{3}\right)_{3}$ have shown inhibitory activity at $25 \mu \mathrm{M}$. Compounds with electron accepting substituents at the $\mathrm{C}-4$ position like $\mathrm{Cl}(\mathbf{8 e}), \mathrm{Br}(\mathbf{8} \mathbf{g})$, and $\mathrm{NO}_{2}(\mathbf{8} \mathbf{h})$ also showed activity at $25 \mu \mathrm{M}$, whereas compounds $\mathbf{8 f}$ (with substituent 4-F) and $8 \mathbf{i}$ (with substituent 4-OH) showed inhibitory activity at $50 \mu \mathrm{M}$. In these meta-substituted compounds, the most potent compound was 9a with a 4-methyl substituent exhibiting activity at $6.25 \mu \mathrm{M}$, which was better than that exhibited by $\mathbf{8 a}$. Compounds $\mathbf{9 b}$ and $\mathbf{9 c}$ with electropositive substituents like $4-\mathrm{OCH}_{3}$ and $3,4-\mathrm{OCH}_{3}$ showed inhibitory activities at $12.5 \mu \mathrm{M}$, both being better than compound $\mathbf{8 b}$ and $\mathbf{8 c}$ with activity at $25 \mu \mathrm{M}$. Compounds $9 \mathrm{~d}, 9 \mathrm{e}, 9 \mathrm{~g}$, and $9 \mathrm{~h}$ displayed activities at $25 \mu \mathrm{M}$, which was similar to activities shown by $\mathbf{8 d}, \mathbf{8 e}, \mathbf{8 g}$, and $\mathbf{8 h}$. Compounds $9 \mathbf{f}$ and $\mathbf{9 i}$ with electronegative substituents 4-F and 4-OH showed better activities at $25 \mu \mathrm{M}$ when compared with $\mathbf{8 f}$ and $\mathbf{8 i}$. With respect to structure-activity relationship, the study revealed that the nature of carbazide and substituent(s) on the phenyl 
Table 2 Molecular parameters of 7-chloro-4-aminoquinoline based semicarbazones and thiosemicarbazones (6a-i), (7a-i),

$(\mathbf{8 a}-\mathbf{i})$, and $(\mathbf{9 a - i})$

\begin{tabular}{|c|c|c|c|c|c|c|}
\hline Compounds & TPSA & n-rotb & $\mathrm{nON}$ & $\mathrm{nOHNH}$ & miLogp & MW \\
\hline Rule of 5 & - & - & $\leq 10$ & $\leq 5$ & $\leq 5$ & $\leq 500$ \\
\hline $6 a$ & 75.33 & 7 & 5 & 4 & 7.101 & 472 \\
\hline $6 b$ & 84.567 & 8 & 6 & 4 & 6.71 & 488 \\
\hline $6 c$ & 93.801 & 9 & 7 & 4 & 6.299 & 518 \\
\hline $6 d$ & 103.035 & 10 & 8 & 4 & 6.284 & 548 \\
\hline $6 e$ & 75.333 & 7 & 5 & 4 & 7.331 & 492 \\
\hline $6 f$ & 75.333 & 7 & 5 & 4 & 6.817 & 475 \\
\hline $6 \mathrm{~g}$ & 75.333 & 7 & 5 & 4 & 7.462 & 536 \\
\hline $6 \mathrm{~h}$ & 121.157 & 8 & 8 & 4 & 6.612 & 502 \\
\hline $6 i$ & 95.561 & 7 & 6 & 5 & 6.174 & 473 \\
\hline $7 a$ & 92.404 & 6 & 6 & 4 & 6.561 & 455 \\
\hline $7 b$ & 101.638 & 7 & 7 & 4 & 6.169 & 471 \\
\hline $7 c$ & 110.872 & 8 & 8 & 4 & 5.759 & 501 \\
\hline $7 d$ & 120.106 & 9 & 9 & 4 & 5.743 & 532 \\
\hline $7 e$ & 92.404 & 6 & 6 & 4 & 6.79 & 476 \\
\hline $7 f$ & 92.404 & 6 & 6 & 4 & 6.276 & 459 \\
\hline $7 \mathrm{~g}$ & 92.404 & 6 & 6 & 4 & 6.921 & 520 \\
\hline $7 \mathrm{~h}$ & 138.228 & 7 & 9 & 4 & 6.071 & 486 \\
\hline $7 i$ & 112.632 & 6 & 7 & 5 & 5.633 & 457 \\
\hline $8 a$ & 75.333 & 7 & 5 & 4 & 7.077 & 472 \\
\hline $8 b$ & 84.567 & 8 & 6 & 4 & 6.686 & 488 \\
\hline $8 c$ & 93.801 & 9 & 7 & 4 & 6.275 & 518 \\
\hline $8 d$ & 103.035 & 10 & 8 & 4 & 6.26 & 548 \\
\hline $8 e$ & 75.333 & 7 & 5 & 4 & 7.307 & 492 \\
\hline $8 f$ & 75.333 & 7 & 5 & 4 & 6.793 & 475 \\
\hline $8 \mathrm{~g}$ & 75.333 & 7 & 5 & 4 & 7.438 & 536 \\
\hline $8 \mathrm{~h}$ & 121.157 & 8 & 8 & 4 & 6.588 & 502 \\
\hline $8 \mathrm{i}$ & 95.561 & 7 & 6 & 5 & 6.15 & 473 \\
\hline $9 a$ & 92.404 & 6 & 6 & 4 & 6.537 & 455 \\
\hline $9 b$ & 101.638 & 7 & 7 & 4 & 6.145 & 471 \\
\hline $9 c$ & 110.872 & 8 & 8 & 4 & 5.735 & 501 \\
\hline $9 d$ & 120.106 & 9 & 9 & 4 & 5.719 & 532 \\
\hline $9 e$ & 92.404 & 6 & 6 & 4 & 6.766 & 476 \\
\hline gf & 92.404 & 6 & 6 & 4 & 6.252 & 459 \\
\hline $9 g$ & 92.404 & 6 & 6 & 4 & 6.897 & 520 \\
\hline $9 \mathrm{~h}$ & 138.228 & 7 & 9 & 4 & 6.047 & 486 \\
\hline $9 i$ & 112.632 & 6 & 7 & 5 & 5.609 & 457 \\
\hline $\mathrm{INH}$ & 68.013 & 1 & 4 & 3 & -0.969 & 137 \\
\hline Pyra & 68.878 & 1 & 4 & 2 & -0.711 & 123 \\
\hline Cipro & 74.569 & 3 & 6 & 2 & -0.701 & 331 \\
\hline
\end{tabular}

TPSA topological polar surface area, $n$-rotb number of rotatable bonds, $n O N$ number of hydrogen bond acceptors, $\mathrm{nOHNH}$ number of hydrogen bond donors, milogP logarithm of compound partition coefficient between n-octanol and water, $M W$ molecular weight, INH isoniazid, Pyra pyrazinamide, Cipro ciprofloxacin ring greatly affects the antitubercular activity. Thus, the comparative study of thiosemicarbazones and semicarbazones revealed that in general, semicarbazone compounds exhibited better inhibitory activity against Mycobacterium tuberculosis.

In recent years, drug likeness and rule of five models [25-27] are a novel approach for better understanding molecular parameters. The obtained results are showing that most of the synthesized compounds have better predicted value of hydrogen bond acceptor, polar surface area, and hydrogen bond donor. According to Lipinski's rule of five, a biological therapeutic ligand should have number of hydrogen bond accepter atoms of 10 or less $(\mathrm{N}$ or $\mathrm{O}$ ), number of hydrogen bond donor atoms of 5 or less, $\log p$ not more than 5 , and molecular weight (MW) of 500 or less. The values of calculated drug scores revealed that most of the synthesized analogs have positive drug likeness score; interestingly, compound 7c exhibited promising inhibitory activity against Mycobacterium tuberculosis and highest drug likeness score Table 2.

\section{Cytotoxicity activity assay}

The in vitro cytotoxicity of the active compounds (MIC $\leq$ $12.5 \mu \mathrm{M}$ Mycobacterium tuberculosis) was evaluated against normal HDF cell line using 3-(4, 5-dimethylthiazo2-yl)-2,5-diphenyl-tetrazolium bromide (MTT) cell proliferation assay. The graphical representation of the cell growth inhibition by the active compounds at a concentration of $50 \mu \mathrm{M}$ is shown in Table 3. The assay data revealed that none of the active compounds are toxic to the normal cells thus proving the lack of general toxicity.

Table 3 Cytotoxicity of compounds against HDF (human dermal fibroblast) cell line

\begin{tabular}{ll}
\hline Compound & \% cell viability ${ }^{\mathrm{a}}(50 \mu \mathrm{M})$ \\
\hline $6 \mathrm{a}$ & 23.87 \\
$6 \mathrm{~b}$ & 20.81 \\
6d & 23.18 \\
6e & 25.83 \\
$7 \mathrm{a}$ & 20.18 \\
$7 \mathrm{c}$ & 26.39 \\
$7 \mathrm{~d}$ & 19.40 \\
$7 \mathrm{fe}$ & 19.57 \\
$7 \mathrm{~g}$ & 24.35 \\
$7 \mathrm{~h}$ & 27.83 \\
$7 \mathrm{i}$ & 23.28 \\
$8 \mathrm{a}$ & 23.87 \\
$9 \mathrm{a}$ & 20.81 \\
$9 \mathrm{~b}$ & 23.18 \\
$9 \mathrm{c}$ & 25.83 \\
\hline
\end{tabular}

${ }^{a}$ Each experiment was independently performed three times, and data are shown as means SD 


\section{Conclusion}

In conclusion, a series of 7-chloro-N-phenylquinolin-4amine derivatives (6a-i), (7a-i), (8a-i), and (9a-i) have been designed and synthesized. All synthesized derivatives underwent in vitro antitubercular evaluation against Mycobacterium tuberculosis $\mathrm{H}_{37} \mathrm{Rv}$ (MTB), and selected analogs were screened for cytotoxicity against normal HDF cell line by MTT cell proliferation assay. The results displayed that among the tested compounds, the most potent antitubercular activity was displayed by $7 \mathrm{c}$ and $7 \mathrm{~g}$ with $\mathrm{MIC}=1.56 \mu \mathrm{M}$ and with low cytotoxicity. Thus, it can be concluded that 7-chloro-N-phenylquinolin-4-amines could be a promising approach for the design of new MTB inhibitory agents.

\section{Additional file}

Additional file 1. Supplementary material.

\section{Abbreviations}

DOTS: Directly observed therapy short-course; INH: Isoniazid; MIC: Minimum inhibitory concentration; MTB: Mycobacterium tuberculosis; MTT: 3-(4, 5dimethylthiazo-2-yl)-2,5-diphenyl-tetrazolium bromide; PZA: Pyrazinamide; RIF: Rifampicin; TB: Tuberculosis

\section{Acknowledgements}

The authors are grateful to Principal, KLE College of Pharmacy, Belagavi, for providing necessary facilities for the research. The authors are also grateful to the NMR Research center, IISC, Bangalore, India, for providing the spectral data.

\section{Authors' contributions}

All authors have read and approved the manuscript. SA designed and outlined and contributed to the characterization of the study. KK, SK, RK, and PS managed the synthetic application. MP, SA, and SS contributed to the analytical work.

\section{Funding}

No funding was obtained for this study.

\section{Availability of data and materials}

All the data generated and analyzed during the study are included in the manuscript.

\section{Ethics approval and consent to participate}

Not applicable

\section{Consent for publication}

Not applicable

\section{Competing interests}

The authors declare that they have no competing interests.

\section{Author details}

${ }^{1}$ Department of Pharmaceutical Chemistry, KLE College of Pharmacy, Belagavi, KLE Academy of Higher Education and Research, Belagavi, Karnataka 590 010, India. ²Department of Pharmacognosy and Phytochemistry, KLE College of Pharmacy, Belagavi, KLE Academy of Higher Education and Research, Belagavi, Karnataka 590 010, India. ${ }^{3}$ Dr. Prabhakar Kore Basic Science Research Center, KLE Academy of Higher Education and Research, Belagavi, Karnataka 590 010, India.
Received: 5 October 2019 Accepted: 27 December 2019

Published online: 16 January 2020

\section{References}

1. World Health Organization. Global Tuberculosis Report 2018 and Global Tuberculosis Report (2018) https://www.who.int/tb/publications/global_ report/en/. Accessed 15 July 2019

2. Bass JB, Farer LS, Hopewell PC, Obrien R, Jacobs RF, Ruben F, Snder DE, Thornton G (1994) Treatment of tuberculosis and tuberculosis infection in adults and children. American Thoracic Society and the centers for Disease Control and Prevention. Am J Respir Crit Care Med 149:1359-1374. https:// doi.org/10.1164/ajrccm.149.5.8173779

3. Davies PDO, Yew WW (2003) Recent developments in treatment of tuberculosis. Expert Opin Investig Drugs 12:1297-1312. https://doi.org/10. 1517/13543784.12.8.1297

4. Tiberi S, Munoz-Torrico M, Duarte R, Dalcolmo M, D'Ambrosio L, Migliori GB (2018) New drugs and perspectives for new anti-tuberculosis regimens. Pulmonol 24:86-98. https://doi.org/10.1016/j.rppnen.2017.10.009

5. Ferrer R, Lobo G, Gamboa N, Rodrigues J, Abramjuk C, Jung K, Lein M, Charris JE (2009) Synthesis of ((7-chloroquinolin-4-yl)amino)chalcones: potential antimalarial and anticancer agents. Sci Pharm 77:725-741. https:// doi.org/10.3797/scipharm.0905-07

6. Kumar A, Srivastava K, Kumar SR, Siddiqi MI, Puri SK, Sexena JK, Chauhan MS (2011) 4-anilinoquinoline triazines: a novel class of hybrid antimalarial agents. Eur J Med Chem 46:676-690. https://doi.org/10.1016/j.ejmech.2010. 12.003

7. Lu CM, Chen YL, Chen HL, Chen CA, Lu PJ, Yang CN (2010) Synthesis and antiproliferative evaluation of certain indolo(3,2-c)quinoline derivatives. Bio Org Med Chem 18:1948-1957. https://doi.org/10.1016/j.bmc.2010.01.033

8. Abonia R, Insuasty D, Castillo J, Insuasty B, Quiroga J, Nogueras M, Cobo J (2012) Synthesis of novel quinoline-2-one based chalcones of potential antitumor activity. Eur J Med Chem 57:29-40. https://doi.org/10.1016/j.ejmech. 2012.08.039

9. Chia EW, Pearce AN, Berridge MV, Larsen L, Perry NB, Sansom CE, Godfrey CA, Hanton LR, Lu GL, Walton M, Denny WA, Webb VL, Copp BR, Harper JL (2002) Synthesis and anti-inflammatory structure-activity relationships of thiazine-quinoline-quinones: inhibitors of the neutrophil respiratory burst in a model of acute gouty arthritis. Bioorg Med Chem 16:9432-9442. https:// doi.org/10.1016/j.bmc.2008.09.052

10. Zarghi A, Ghodsi R, Azizi E, Daraie B, Hedayati M, Dadrass OG (2009) Synthesis and biological evaluation of new 4-carboxyl quinoline derivatives as cyclooxygenase-2 inhibitors. Bio Org Med Chem 17:5312-5317. https:// doi.org/10.1016/j.bmc.2009.05.084

11. Jablonski JJ, Basu D, Engel DA, Geysen HM (2012) Design, synthesis, and evaluation of novel small molecule inhibitors of the influenza virus protein NS1. Bio Org Med Chem 20:487-497. https://doi.org/10.1016/j.bmc.2011.10. 026

12. Chen YL, Tang J, Kesler MJ, Sham YY, Vince R, Geraghty RJ, Wang Z (2012) The design, synthesis and biological evaluations of C-6 or C-7 substituted 2hydroxyisoquinoline-1,3-diones as inhibitors of hepatitis C virus. Bio Org Med Chem 20:467-479. https://doi.org/10.1016/j.bmc.2011.10.058

13. Garudachari B, Satyanarayana MN, Thippeswamy B, Shivakumar CK Shivananda HN, Hegde G, Isloor AM (2012) Synthesis, characterization and antimicrobial studies of some new quinoline incorporated benzimidazole derivatives. Eur J Med Chem 54:900-906. https://doi.org/10.1016/j.ejmech. 2012.05.027

14. Eswaran S, Adhikari AV, Shetty NS (2009) Synthesis and antimicrobial activities of novel quinoline derivatives carrying 1,2,4-triazole moiety. Eur J Med Chem 44:4637-4647. https://doi.org/10.1016/j.ejmech.2009.06.031

15. Musiol R, Jampilek J, Buchta V, Silva L, Niedbala H, Podeszwa B, Palka A, Maniecka KM, Oleksyn B, Polanski J (2006) Antifungal properties of new series of quinoline derivatives. Bioorg Med Chem 14:3592-3598. https://doi. org/10.1016/j.bmc.2006.01.016

16. Fiorito J, Saeed F, Zhan SA, Feng Y, Francis YL, Rao S, Thakkar DM, Deng SH, Landry DW, Arancio O (2013) Synthesis of quinoline derivatives: discovery of a potent and selective phosphodiesterase 5 inhibitor for the treatment of Alzheimer's disease. Eur J Med Chem 60:285-294. https://doi.org/10.1016/j. ejmech.2012.12.009

17. Marella A, Tanwar OP, Shah R, Ali MR, Srivastava S, Akhter M, Shaquiquzzaman M, Alam MM (2013) Quinoline: a versatile heterocyclic Saud Pharm J 21:1-12. https://doi.org/10.1016/j.jsps.2012.03.002 
18. Rangappa SK, Siddappa AP (2014) Quinoline: a promising antitubercular target. Biomed Pharmacother 68:1161-1175. https://doi.org/10.1016/j. biopha.2014.10.007

19. Jallapally A, Adbla D, Yogeeswari P, Sriam D, Kantevari S (2014) 2-Butyl-4chloroimidazole based substituted piperazine-thiosemicarbazone hybrids as potent inhibitors of Mycobacterium tuberculosis. Bioorg Med Chem Lett 24: 5520-2524. https://doi.org/10.1016/j.bmcl.2014.09.084

20. Pavan FR, Mira PIS, Leite SRA, Deflon VM, Batista AA, Sato DN, Franzblu SG, Leite CQF (2010) Thiosemicarbazones, semicarbazones, dithiocarbazates and hydrazide/hydrazones: anti-mycobacterium tuberculosis activity and cytotoxicity. Eur J Med Chem 45:1898-1905. https://doi.org/10.1016/j. ejmech.2010.01.028

21. Sharma M, Chaturvedi V, Manju YK, Batnagar S, Shrivastava K, Puri SK, Chauhan PMS (2009) Substituted quinolinyl chalcones and quinolinyl pyrimidines as a new class of anti-infective agents. Eur J Med Chem 44: 2081-2091. https://doi.org/10.1016/j.ejmech.2008.10.011

22. Franzblau SG, Witzig RS, McLaughlin JC, Torres P, Madico G, Hernandez A, Degnan MT, Cook MB, Quenzer VK, Ferguson RM, Gilman RH (1998) Rapid, low-technology MIC determination with clinical mycobacterium tuberculosis isolates by using the microplate alamar blue assay. J Clin Microbiol 36:362-366

23. Alegaon SG, Alagawadi KR, Sonkusare PV, Chaudhary SM, Dadwe DH, Shah AS (2012) Novel imidazo(2,1-b)(1,3,4) thiadiazole carrying rhodanine-3-acetic acid as potential antitubercular agents. Bioorg Med Chem Lett 22:19171921. https://doi.org/10.1016/j.bmcl.2012.01.052

24. Mosmann T (1983) Rapid colorimetric assay for cellular growth and survival: application to proliferation and cytotoxicity assays. J Immunol Methods 65: 55-63. https://doi.org/10.1016/0022-1759(83)90303-4

25. Lipinski CA, Lombardo F, Dominy BW, Feeney PJ (2001) Experimental and computational approaches to estimate solubility and permeability in drug discovery and development settings. Adv Drug Deliv Rev 46:3-26

26. Cheminformatics M Bratislava. Slovak Republic http://www.molinspiration. com/services/properties.html. Accessed 10 July 2019

27. Drug-Likeness and molecular property prediction. http://molsoft.com/ mprop/ Accessed 10 July 2019

\section{Publisher's Note}

Springer Nature remains neutral with regard to jurisdictional claims in published maps and institutional affiliations.

\section{Submit your manuscript to a SpringerOpen ${ }^{\circ}$ journal and benefit from:}

- Convenient online submission

- Rigorous peer review

- Open access: articles freely available online

- High visibility within the field

- Retaining the copyright to your article

Submit your next manuscript at $\boldsymbol{\nabla}$ springeropen.com 